\title{
Arquitectura y anatomía foliar del complejo Polypodium plesiosorum sensu Moran (Polypodiaceae)
}

J. Daniel Tejero-Díez ${ }^{1}$, Silvia Aguilar-Rodríguez ${ }^{1}$, Teresa Terrazas ${ }^{2}$ \& Leticia Pacheco ${ }^{3}$

1. Facultad de Estudios Superiores Iztacala, Universidad Nacional Autónoma de México, Unidad de Morfología y Función, Av. de los Barrios 1, Los Reyes Iztacala, Tlalnepantla 54090, Edo. México, México, Apdo. Postal 314; tejero@unam.mx; siagro@campus.iztacala.unam.mx

2. Instituto de Biología, Universidad Nacional Autónoma de México, Av. Universidad, Coyoacán 04510, México D.F., Apdo. Postal 70-233; tterrazas@ibiologia.unam.mx

3. Depto. Biología, Universidad Autónoma Metropolitana-Iztapalapa, Botánica Estructural y Sistemática Vegetal, Av. San Rafael Atlixco 186, Vicentina, Iztapalapa 09340, México D. F., Apdo. Postal 55-535; pacheco@xanum.uam.mx

Recibido 23-VIII-2009. Corregido 07-I-2010. Aceptado 08-II-2010.

\begin{abstract}
Architecture and leaf anatomy of the Polypodium plesiosorum sensu Moran complex (Polypodiaceae). The Polypodium plesisorum complex is a heterogeneous morphology group. We studied leaf architecture and anatomy of 12 species of this group, in addition to other 23 species of Polypodium $(P$. dulce, P. polypodioides and P. vulgare complex sensu Moran) and related genera (Goniophlebium, Phlebodium, Pleopeltis, Serpocaulon, Synammia), for comparative purposes. Two homogeneous groups of species were established in P. plesiosorum complex. One of them is recognized based on the type of anastomosis of the veins (Type III): Polypodium conterminans (originally considered in the group of $P$. dulce with free venation by Moran), P. hispidulum, P. plesiosorum and P. rhodopleuron. The second group with Polypodium arcanum, P. castaneum, $P$. colpodes, $P$. eatonii, and $P$. flagellare were recognized based on the type of anastomosis of the veins (Type IV) and how vascular strands fuse along the petiole-leaf rachis. Characters studied helped to group the remaining species with other groups outside the complex under study. Rev. Biol. Trop. 58 (3): 955-976. Epub 2010 September 01.
\end{abstract}

Key words: polypodiaceae, Polypodium plesiosorum complex, leaf architecture, leaf anatomy, Mexico.

La arquitectura y la morfo-anatomía foliar de los helechos han sido analizadas con diferentes fines (Wardlaw 1944, Iwatsuki 1962, de la Sota 1963, Tryon 1964, 1970, Lucansky \& White 1974, Kaur 1978, Wagner 1979, Beck et al. 1982, Salgado 1982, White 1984, Saiki et al. 1988, Steeves 1988, Stewart \& Rothwell 1993). En la familia Polypodiaceae, la disposición de las venas ha apoyado el establecimiento de grupos y géneros en los sistemas de clasificación (Presl 1836, Maxon 1916 b, Ching 1940, Copeland 1947); sin embargo, sólo en algunos estudios se discute la relación entre los patrones de venación y los aspectos taxonómicos o evolutivos (Christensen 1928, Tindale 1960, de la Sota 1963, 1966, Hetterscheid \& Hennipman
1984, Mitsuta 1984, Hensen 1990, Rödl-Linder 1990). Sobre la anatomía del pecíolo-raquis, de la Sota (1966) y Rödl-Linder (1990) describen la forma en que se organizan los cordones vasculares en los taxa que estudiaron y sólo Ching (1933) evidencia la utilidad de estos cordones como elementos de valor taxonómico en Polypodiaceae de China para discernir grupos. Por otra parte, Sen \& Hennipman (1981) abordaron los tipos de aparatos estomáticos en 32 especies de Polypodium (s.l.). Sin embargo, son escasos los trabajos referentes a la anatomía de la lámina.

La ubicación taxonómica de Polypodium L. es problemática (Hennipman 1986, Wagner 1986, Hennipman et al. 1990), a partir de él se han segregado otros como Campyloneurum C. 
Presl., Goniophlebium C. Presl, Marginariopsis C. Chr., Microsorium Link, Pecluma M. G. Price, Phlebodium (R. Br.) J. Sm., Pleopeltis Humb. \& Bonpl. ex Willd., Serpocaulon A. R. Sm. y Synammia C. Presl (Evans 1969, Gómez 1975, Wagner \& Wagner 1975, Anthony \& Schlepe 1985, Mickel \& Beitel 1987, Bosman 1991, Widham 1993, Smith et al. 2006, Otto 2007, Salino et al. 2008, Tejero-Díez et al. 2009). Además, con base en caracteres morfológicos, se han definido varios complejos en Polypodium (Maxon 1903, 1916 a-c, Weatherby $1922,1939,1947$, Lloyd \& Lang 1964, de la Sota 1965, 1966, Tryon \& Tryon 1982, Price 1983, Hennipman et al. 1990, Hensen 1990, Windham 1993, Moran 1995, Neuroth 1996). En este siglo, caracteres moleculares o morfoanatómicos analizados por el método cladista, están esclareciendo algunas de las relaciones de parentesco entre las diferentes jerarquías taxonómicas de Polypodiaceae (Smith et al. 2000, Ranker et al. 2004, Schneider et al. 2004, Tejero-Díez 2005, Schneider et al. 2004, 2006, Otto 2007, Schuettpelz \& Pryer 2007) y están propiciando una clasificación más sólida (Smith et al. 2006).

Polypodium (sensu stricto) se distingue por tener tallo rizomatoso alargado y ramificado, escamas del rizoma con celdillas opacas, hojas con pecíolo surcado en la superficie adaxial y lámina pinnatisecta a 1-pinnada con los segmentos adnatos al raquis, esporas monoletes con la superficie típicamente verrugosa generalmente sin perisporio y número cromosómico conocido $n=37$. El taxón así definido considera la reciente segregación de Serpocaulon (Smith et al. 2006) e incluye 70 a 75 especies, la mayoría con distribución en el continente americano y algunas en el Viejo Mundo.

El conjunto de especies de Polypodium carentes de escamas laminares, incluidas por Tryon \& Tryon (1982) en el grupo de Polypodium plesiosorum Kunze y por Moran (1995) en los grupos $P$. plesiosorum y $P$. dulce Poir., han recibido hasta el momento poca atención taxonómica. Smith (1981), Stolze (1981) y Mickel \& Beitel (1988) indican que subsisten problemas de circunscripción de subgrupos y de especies. Moran (1995) consideró como "grupo $P$. plesiosorum" a: $P$. colpodes Kunze $(=P$. fuscopetiolatum A. R. Sm.), P. eatonii Baker, P. flagellare H. Christ, P. fraternum Schltdl. \& Cham., P. hispidulum Bartlett, P. kunzeanum C. Chr., P. plesiosorum Kunze, P. pleurosorum Kunze ex Mett., P. rhachipterygium Liebm. y $P$. rhodopleuron Kunze. Además, Tejero-Díez \& Pacheco (2004) incluyeron por definición a $P$. arcanum Maxon y $P$. castaneum Maxon ex Tejero, ya que el primero se encuentra fuera del área de Mesoamérica y el segundo es de reciente nombramiento. En el trabajo original (Tejero-Díez 2005), por definición, se incluyó a Synammia feuillei (Bertero) Copel. (=Polypodium feuillei Bertero).

En este estudio se analiza la arquitectura y anatomía foliar de las especies del complejo $P$. plesiosorum (sensu Moran 1995) y se compara con especies de otros complejos y géneros cercanos que crecen en México y Centroamérica, con el propósito de identificar caracteres que permitan apoyar el reconocimiento de un grupo P. plesiosorum homogéneo y reubicar taxonómicamente al resto de las especies.

Todas las especies revisadas habitan en México, excepto Polypodium kunzeanum y Synammia feuillei que presentan una distribución sudamericana.

\section{MATERIALES Y MÉTODOS}

Se estudiaron las 12 especies reconocidas para el complejo de Polypodium plesiosorum (sensu Moran 1995) y otras 23 de Polypodium y especies representantes de géneros segregados con fines comparativos (Apéndice I).

Arquitectura foliar: Se consultaron ejemplares depositados en 27 herbarios (Tejero-Díez 2005). Las hojas se estudiaron directamente de material herborizado o fijado en FAA (formol, ácido acético glacial y alcohol etílico al $70 \%$, 5:5:90). Los patrones de venación se observaron directamente al microscopio estereoscópico por transiluminación. Sólo en aquellos casos, en que el grosor del mesofilo de la lámina o el indumento de la misma no permitieron ver el 
patrón de disposición de las venas, se practicó la técnica de diafanizado, la cual consistió en sumergir al segmento o parte de la lámina en una solución de hidróxido de potasio al $2 \%$ con hipoclorito de sodio diluido al 5\% durante dos a cuatro días. Los términos y conceptos sobre los caracteres de las hojas y las venas se tomaron de Wagner (1979) y Rödl-Linder (1990).

Anatomía: Los caracteres anatómicos se estudiaron en uno a cinco individuos recolectados en el campo, con ejemplares de referencia para herbario (Apéndice I). El material biológico recolectado se fijó en solución FAPA (formol $40 \%$, ácido acético glacial, ácido propiónico y alcohol al 50\%, 5:2.5:2.5:90) durante un mes; posteriormente se conservó en solución GAA (glicerina-alcohol-agua, 1:2:3) hasta su procesamiento en el laboratorio. Para algunas especies el material se obtuvo a partir de ejemplares de herbario y, los fragmentos de pecíolos, raquis y/o pinnas removidos se hirvieron en una solución de glicerina-agua 1:1 para rehidratarlos y posteriormente se fijaron en solución FAPA.

Aparato estomático. Se obtuvieron réplicas negativas de la superficie epidérmica abaxial, mediante la aplicación de un barniz compuesto de celulosa acética y resina (Payne 1968), las cuales se montaron en gelatina glicerinada para su observación en el microscopio óptico. Los tipos de aparatos estomáticos se describieron de acuerdo a Sen \& Hennipman (1981), excepto el tipo diacítico de Fryns-Claessens \& van Cotthem (1973). Con la finalidad de evaluar el predominio de ciertos tipos de aparatos en las especies, se observaron dos o tres muestras, se hicieron 50 conteos por muestra y se calculó el porcentaje.

Preparaciones histológicas. Para lograr la descripción histológica de los ejes y láminas, las muestras fijadas en GAA, se deshidrataron por medio de una serie de cambios de concentración creciente de etanol y ter-butanol (Johansen 1940). A partir del último cambio, se agregó en el lapso de dos o tres día pequeñas cantidades de parafina hasta la proporción 1:1 parafina/ter-butanol; posteriormente se dejó evaporar el ter-butanol en una estufa y las muestras se incluyeron en un bloque de parafina. De los bloques, se obtuvieron secciones transversales del pecíolo, raquis y segmento laminar de 20-25 $\mu \mathrm{m}$ de grosor; se tiñeron con safranina-verde rápido (Johansen 1940) y se montaron en portaobjetos con resina sintética. Las laminillas se depositaron como referencia en las colecciones de los herbarios de la Facultad de Estudios Superiores Iztacala (IZTA) y Nacional de México (MEXU), ambos en la Universidad Nacional Autónoma de México.

Cordones vasculares. El número, tipo $\mathrm{y}$ forma en que se fusionan o dividen los cordones vasculares a lo largo del eje foliar, fueron observados al efectuar cortes transversales a mano alzada en la parte basal (tanto en el filopodio como en la parte inmediata posterior a la articulación), media y distal del pecíolo; así como en la base (entre el segundo y tercer par de segmentos), la parte media y la distal del raquis. Las secciones se montaron en gelatina-safranina (AguilarRodríguez 1998). Siempre que fue posible se analizaron varias muestras de una especie dada, o incluso pecíolos con diferentes grosores del mismo ejemplar. La manera en que se disponen los cordones vasculares en el eje de la hoja sigue en parte el concepto de Rödl-Linder (1990); se analizaron dos caracteres básicos: el tamaño y la forma de fusión o división de los cordones a lo largo del eje. Para ilustrar las descripciones se tomaron fotografías y se realizaron dibujos con cámara clara a partir de un microscopio óptico Nikon Labophoto-2.

\section{RESULTADOS}

\section{Arquitectura foliar}

\section{Forma y división de la lámina}

En el grupo de Polypodium plesiosorum se reconocieron cuatro tipos de láminas: pinnatisecta, pinnatipartida, semipinnada y 1-pinnada; la primera se presenta en siete especies, siendo basipinnada en dos de ellas (Cuadro 1). En las especies que no pertenecen al complejo se encontraron los mismos tipos, además de láminas simples y pinnatífidas. 
CUADRO 1

Tipos de división, segmento apical y margen de la lámina en especies del grupo Polypodium plesiosorum (sensu Moran 1995). subcom=subcomforme. * Especies incluidas por definición ya que se distribuyen fuera del área de estudio de la Flora Mesoamericana o han sido descritas recientemente

TABLE 1

Blade characteristics in species of Polypodium plesiosorum group (sensu Moran 1995). Subcom=subconform.

* Species included by definition since they are distributed out of the area of study of the Mesoamerican flora or have been described recently

$\begin{array}{llcc}\text { Polypodium /Lámina } & \text { División } & \text { Segmento apical } & \text { Margen } \\ \text { *P. } \text { arcanum } & \text { Pinnatisecta } & \text { graduado/subcom. } & \text { crenado } \\ \text { *P. castaneum } & \text { Pinnatisecta/basipinnada } & \text { graduado/subcom. } & \text { crenado } \\ \text { P. colpodes } & \text { Pinnatisecta/basipinnada } & \text { graduado/subcom. } & \text { crenado } \\ \text { P. eatonii } & \text { pinnatisecta y/o pinnatipartida } & \text { graduado/subcom. } & \text { entero } \\ \text { P. flagellare } & \text { Semipinnada } & \text { graduado/subcom. } & \text { crenado } \\ \text { P. fraternum } & \text { Pinnada } & \text { conforme } & \text { crenado } \\ \text { P. hispidulum } & \text { Pinnatisecta } & \text { graduado/subcom. } & \text { crenado } \\ \text { P. } \text { kunzeanum } & \text { Pinnada (pinnas articuladas al raquis) } & \text { conforme } & \text { crenado } \\ \text { P. plesiosorum } & \text { Pinnatisecta } & \text { graduado/subcom. } & \text { crenado } \\ \text { P. pleurosorum } & \text { Pinnada } & \text { conforme } & \text { crenado } \\ \text { P. rhachipterygium } & \text { Pinnatipartida } & \text { graduado/subcom. } & \text { entero } \\ \text { P. rhodopleuron } & \text { Pinnatisecta } & \text { graduado/subcom. } & \text { crenado }\end{array}$

Entre las especies del grupo estudiado los segmentos basales son tan largos o más que los segmentos medios, pero presentan una unión al eje muy disímil: Polypodium rhachipterygium es largamente decurrente, $P$. eatonii y $P$. kunzeanum, pueden o no tener lóbulos, en $P$. fraternum son estrechamente sésiles y en el resto de las especies varían entre escavados a anchamente sésiles y levemente decurrentes en su base. La mayor parte de las especies presentan los segmentos basales transversales al eje, sin embargo, en P. hispidulum, P. plesiosorum y $P$. rhodopleuron tienden a estar reflejos.

De acuerdo a la disposición de los segmentos de la lámina en su porción distal, se distinguen tres tipos de ápices en todas las especies estudiadas: graduado, conforme y subconforme. Nueve especies del grupo bajo estudio presentan ápice graduado o subconforme, pero las especies con lámina pinnada sólo tienen ápice conforme (Cuadro 1).

Por lo anterior, el contorno de la lámina en las especie del grupo estudiado es en general deltado a ovado-lanceolado en diferentes proporciones, donde aparentemente cada especie tiene su propia particularidad.

El margen de la lámina es generalmente crenado en el grupo estudiado; con excepción de $P$. eatonii y $P$. rhachipterygium en donde es entero (Cuadro 1).

\section{Disposición de las venas en la lámina}

El sistema de venas secundarias (vs) de cada segmento es catádromo. En las venas terciarias, la basal siempre es fértil (vf) y en ocasiones también las terciarias subsecuentes. En las especies estudiadas los patrones de fusión, división o reducción de las venas terciarias o de orden superior fértiles o estériles (ves) se caracterizan por presentar una variación gradual que va desde un sistema de venas abiertas o libres (Figs. 1A, B) hasta anastomosadas o cerradas (Figs. 1C-H). Las venas anastomosadas se caracterizan por formar aréolas que presentan varios niveles de complejidad al conformar distintos tipos de polígonos angulares (también llamados goniofleboides), así como por la presencia o no de una 
A
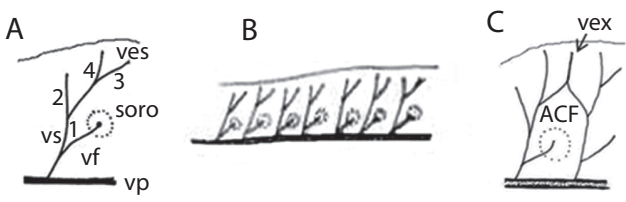

D

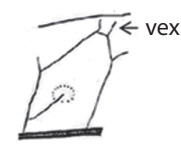

$\mathrm{E}$

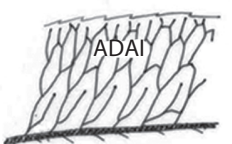

$\mathrm{F}$

AFA

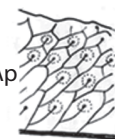

G

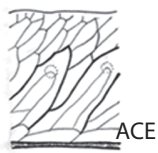

$\mathrm{H}$

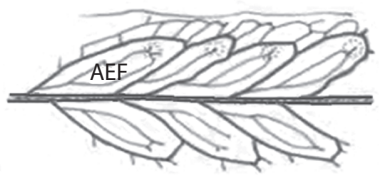

$10 \mathrm{~mm}$

Fig. 1. Patrones de venación. Los tipos indican una variación gradual desde un sistema de venas abiertas hasta las anastomosadas que forman aréolas: A-B. Venas abiertas. A. Tipo I. B. Tipo II. C-H. Venas anastomosadas. C. Tipo III. D. Tipo IV. E. Tipo V. F. Tipo VI. G. Tipo VII. H. Tipo VIII.

$\mathrm{ACE}=$ aréola costal estéril; $\mathrm{ACF}=$ aréola costal fértil; $\mathrm{ADAl}=$ fila de aréolas discoides alternas; $\mathrm{AFAp}=$ aréolas fértiles apiladas; $\mathrm{AEF}=$ aréola elíptica fértil; ves=vena estéril; vex=vena excurrente; $v f=$ vena fértil; $v p=v e n a$ primaria; vs $=$ vena secundaria.

Fig. 1. Venation patterns. The types show a gradual variation from a system of free veins to anastomosing veins which form areoles: A-B. Open veins. A. Type I. B. Type II. C-H. Anastomosed veins. C. Type III. D. Type IV. E. Type V. F. Type VI. G. Type VII. H. Type VIII. ACE $=$ costal sterile areole; $\mathrm{ACF}=$ costal fertile areole; $\mathrm{ADAl}=$ disc-shaped alternates areoles row; $\mathrm{AFAp}=$ fertile piled up areoles; $\mathrm{AEF}=$ elliptical fertile areole; ves $=$ sterile vein; vex=excurrent vein; $v f=$ fertile vein; $v p=$ primary vein; vs $=$ secondary vein.

vena fértil en su interior. A su vez, la vena fértil es simple en los primeros niveles de complejidad, pero llega a tener varios patrones de ramificación en los niveles más altos. El análisis comparativo de las especies del grupo P. plesiosorum y de complejos o géneros relacionados estudiados, permitió establecer ocho tipos de patrones de venación y distribución en los segmentos de la lámina foliar (Cuadro 2).

Tipo I. Venas terciarias libres (Fig. 1A). El tipo I es característico del grupo de $P$. dulce. En el grupo estudiado, el $1 \%$ de los ejemplares revisados de P. plesiosorum presentaron este tipo. En $P$. fraternum el $60 \%$ o más de los ejemplares presentan venas libres y el resto son del Tipo III por lo que el carácter en esta especie es dimorfo. $P$. rhachipterygium presenta algunas poblaciones con este tipo de venas. En todas las especies del complejo en estudio, la porción distal de cada segmento muestra venación libre. Esta proporción es mayor (hasta $3 / 4$ partes del segmento) en Polypodium hispidulum, P. plesiosorum y P. rhodopleuron. La proporción de venas libres en el segmento es menor (hasta $1 / 2$ del segmento) en Polypodium arcanum, $P$. castaneum, $P$. colpodes, $P$. eatonii y $P$. flagellare y es prácticamente inexistente (menos de la $1 / 4$ parte del segmento) en $P$. pleurosorum y P. kunzeanum.

Tipo II. Semejante al tipo I, pero con reducción de las venas terciarias en número o longitud (Fig. 1B). Esta disposición no se observó en las especies del grupo de P. plesiosorum, pero lo presentaron Pecluma dispersa (A. M. Evans) M. G. Price, P. martensii Mett. y P. furfuraceum Schltdl. et Cham.

Tipo III. Venación terciaria anastomosada que forma una hilera a cada lado de la vena primaria (también denominada costa en este trabajo) de aréolas pentagonales que encierran a la vena fértil (ACF) y presentan una vena excurrente externa (vex) (Fig. 1C). Se observó en P. plesiosorum, P. rhodopleuron, P. hispidulum y $P$. rhachipterygium aunque en esta última especie no en todos lo ejemplares examinados. En $P$. conterminans Liebm., del grupo de $P$. dulce, aproximadamente el $80 \%$ de los ejemplares presentan también este tipo. En estas especies, la aréola es alargada, de la misma forma que aquellas que se forman accidentalmente en especies de los grupos de $P$. dulce y $P$. vulgare L. La aréola pentagonal también es 
CUADRO 2

Distribución y tipo de venas en los segmentos de la lámina foliar en el grupo de Polypodium plesiosorum (Moran 1995)

TABLE 2

Distribution and type of veins in segments of the blade in the Polypodium groups (Moran 1995)

\begin{tabular}{|c|c|c|c|c|c|c|}
\hline Tipos & $\begin{array}{l}\text { Venas terciarias } \\
\text { o superiores }\end{array}$ & $\begin{array}{l}\text { Aréola costal } \\
\text { (fértil) }\end{array}$ & $\begin{array}{l}\text { Vena } \\
\text { fértil }\end{array}$ & $\begin{array}{l}\text { Alimentación } \\
\text { al soro }\end{array}$ & $\begin{array}{l}\text { Aréola } \\
\text { fértil } \\
\text { secundaria }\end{array}$ & $\begin{array}{l}\text { Aréolas } \\
\text { discoidales } \\
\text { marginales }\end{array}$ \\
\hline I & libres desarrolladas & ausente & simple & simple & ausente & ausente \\
\hline II & libres reducidas & ausente & simple & simple & ausente & ausente \\
\hline III & anastomosadas & $\begin{array}{l}\text { pentagonal alargada } \\
\text { o isodiamétrica, una } \\
\text { hilera }\end{array}$ & simple & simple & ausente & ausente \\
\hline IV & anastomosadas & $\begin{array}{l}\text { pentagonales/ } \\
\text { hexagonales, una } \\
\text { hilera }\end{array}$ & simple & simple & ausente & $\begin{array}{l}\text { ausente o } \\
\text { irregulares }\end{array}$ \\
\hline $\mathrm{V}$ & anastomosadas & $\begin{array}{l}\text { poligonales (siete a } \\
\text { doce ángulos), una } \\
\text { hilera }\end{array}$ & simple & simple & ausente & $\begin{array}{l}\text { con una o más } \\
\text { hileras }\end{array}$ \\
\hline VI & anastomosadas & $\begin{array}{l}\text { pentagonales } \\
\text { isodiamétricas } \\
\text { varias hileras } \\
\text { apiladas }\end{array}$ & simple & simple & ausente & $\begin{array}{l}\text { ausente o } \\
\text { irregulares }\end{array}$ \\
\hline VII & anastomosadas & $\begin{array}{l}\text { poligonales (siete a } \\
\text { doce ángulos), una } \\
\text { hilera }\end{array}$ & $\begin{array}{l}\text { dividida en } \\
\text { ángulo subrecto }\end{array}$ & dos ramas & presente & $\begin{array}{l}\text { con una o más } \\
\text { hileras }\end{array}$ \\
\hline VIII & anastomosadas & $\begin{array}{l}\text { poligonal con más } \\
\text { de doce ángulos, } \\
\text { una hilera }\end{array}$ & $\begin{array}{l}\text { dividida } \\
\text { primariamente en } \\
\text { ángulo agudo }\end{array}$ & $\begin{array}{l}\text { por la unión de } \\
\text { varias venas } \\
\text { (compital) }\end{array}$ & presente & con una hilera \\
\hline
\end{tabular}

característica de Serpocaulon A. R. Sm. aunque el pentágono formado es isodiamétrico.

Tipo IV. Venación terciaria y cuaternaria anastomosada que forman una hilera a cada lado de la vena primaria de aréolas pentagonales y/o hexagonales que encierran a la vena fértil y presentan dos venas excurrentes externas (vex) (Fig. 1D). Se observó en $P$. arcanum, $P$. castaneum, $P$. colpodes, $P$. eatonii y $P$. flagellare.

Tipo V. Venación cuaternaria o de orden superior anastomosadas que forman una hilera a cada lado de la vena primaria de aréolas poliangulares (de ocho o más ángulos). A su vez, las venas marginales se vuelven a anastomosar formando una o más hileras de aréolas discoides alternas a las costales (ADAl), que generalmente son estériles por carecer de venilla interna (Fig. 1E). Se observó en P. eatonii, $P$. kunzeanum y $P$. pleurosorum del grupo de interés, así como en Phlebodium pseudoaureum (Cav.) Lellinger, P. xhemipinnatum Tejero, Mickel \& A.R. Sm. y en varias especies del grupo de Polypodium polypodioides (L.) Watt.

Tipo VI. Aréolas fértiles secundarias apiladas (AFAp), que se producen por anastomosis sucesivas de las venas terciarias, donde la vena excurrente a su vez queda encerrada y produce un soro en el ápice. (Fig. 1F). Son típicas de algunas especies de Serpocaulon como $S$. triseriale (Sw.) A. R. Sm. Excepcionalmente se observaron aréolas apiladas infértiles en Polypodium plesiosorum y P. rhodopleuron del grupo bajo estudio. 
Tipo VII. La vena interna fértil de las aréolas costales se dividen produciendo venas de cuarto o quinto orden que forman una aréola secundaria costal estéril (ACE) y una alimentación dos ramas del soro (Fig. 1G). Se observó en Phlebodium pseudoaureum y en $P h$. Xhemipinnatum es variable; en Polypodium pleurosorum del grupo de interés es casual en las láminas de algunos ejemplares.

Tipo VIII. La vena interna fértil de las aréolas costales se divide y fusiona nuevamente formando una aréola elíptica (AEF) (Fig. 1H). En Pleopeltis angusta o P. mexicana a su vez, pueden dividirse y anastomosarse nuevamente para formar un patrón complejo de areolillas internas. El soro se encuentra en la confluencia de varias venillas (soro compital). Las venas estériles se organizan en las distintas especies observadas del grupo de Polypodium polypodioides de acuerdo con los tipos I, III, IV y V. Este tipo no se observó en las especies del complejo de P. plesiosorum.

\section{Anatomía}

\section{Peciolo-raquis}

En las especies del grupo de Polypodium plesiosorum estudiadas, la base del pecíolo esta articulado por medio de una banda de abscisión (Figs. 2A, B) que permite a la hoja desprenderse al iniciar la temporada de sequía, mientras que un remanente peciolar, el filopodio, permanece unido al rizoma. En sección transversal (Fig.
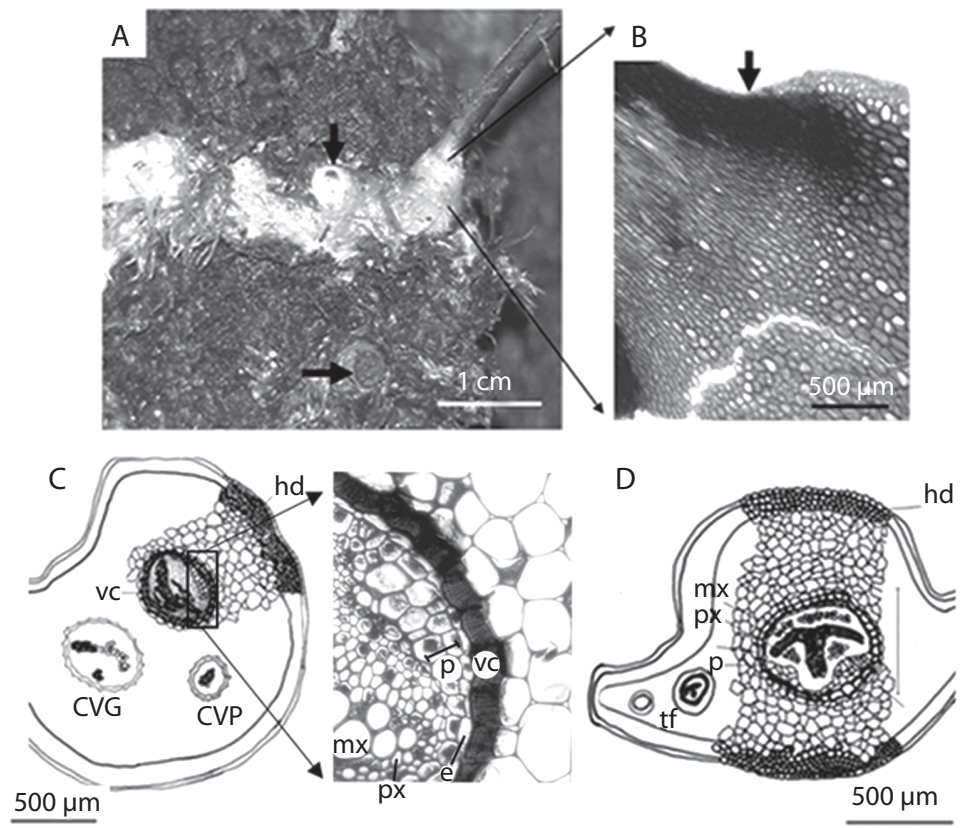

Fig. 2. Filopodio-pecíolo y raquis. A, B. morfología y anatomía del filopodio, vistas superficial y longitudinal respectivamente. C. Corte transversal en la base del pecíolo en Polypodium colpodes. (D. Tejero Díez 4270 IZTA) (130 $\mathrm{X}$ y recuadro a $400 \mathrm{X}$ ). D. Corte transversal en la base del eje raquis en Polypodium colpodes. (D. Tejero Diez 4270 IZTA) $(200 \mathrm{X}) . \mathrm{CVG}=$ cordones vasculares grandes; $\mathrm{CVP}=$ cordones vasculares pequeños; e=endodermis; hd=hipodermis; $\mathrm{mx}=$ metaxilema; $\mathrm{p}=$ periciclo; $\mathrm{px}=$ protoxilema; $\mathrm{tf}=$ trazas foliares; $\mathrm{vc}=$ =vaina circumendodermal. Flechas=zona de abscisión.

Fig. 2. Phyllopodium-petiole and rachis. A, B. Morphology and anatomy of phyllopodium. C. Transverse section of petiole base in Polypodium colpodes (D. Tejero Diez 4270 IZTA) (130 X and square 400 X). D. Transverse section in the base of the rachis axis in Polypodium colpodes. (D. Tejero Diez 4270 IZTA) $(200 \mathrm{X}) . \mathrm{CVG}=$ large vascular strands; $\mathrm{CVP}=\mathrm{small}$ vascular strands; hd=hypodermis; $\mathrm{vc}=$ circumendodermal sheath; $\mathrm{e}=$ =ndodermis; $\mathrm{p}=$ pericycle; $\mathrm{mx}=\mathrm{metaxylem}$; $\mathrm{px}=\mathrm{protoxylem}$; $\mathrm{tf}=$ leaf trace. Arrows=abscission zone. 
2C), el pecíolo es redondeado, con tendencia a aplanarse o acanalarse una o dos veces en la superficie adaxial. En la superficie del canal se observan tricomas simples, cateniformes o capitados, sobre todo en dirección acrópeta, en $P$. colpodes, $P$. hispidulum y $P$. plesiosorum. La epidermis es simple y presenta una cutícula gruesa. Debajo de la epidermis se diferencia una hipodermis (hd) constituida por tres a seis hileras de fibras con engrosamiento de la pared de mediano a muy notable, que en ocasiones llega a obliterar el lumen. Esta banda de esclerénquima forma un cilindro continuo a lo largo del pecíolo excepto en la parte distal y en el raquis, que suele interrumpirse a la altura de las alas foliares (Fig. 2D). Bajo la hipodermis, existen de cinco a ocho hileras de células parenquimáticas con forma isodiamétrica que ocasionalmente contienen granos de almidón. En Polypodium kunzeanum se observan cristales prismáticos y fibras dispersas en el parénquima. En el tejido parenquimático se encuentran embebidos (uno) dos cordones vasculares grandes (CVG) en posición adaxial y uno a 13 pequeños (CVP), formando un arco en la posición abaxial; cada uno de los cordones está rodeado por una hilera de células, cuya pared tangencial interna presenta un engrosamiento sobresaliente de celulosa con taninos, denominada vaina circumendodermal (vc) (V. Hernández, T. Terrazas, K. Mehltreter \& G. Ángeles, en prep.). En el interior de cada vaina se desarrolla la endodermis (e) formada por una capa de células (Fig. 2C) y el periciclo (p) de una capa celular excepto en la región externa del meristele. En la porción central del cordón vascular se dispone el tejido xilemático que tiene forma de arco ("C") con el protoxilema exarco (Fig. 2C). Entre el periciclo y los elementos del xilema se encuentra el tejido floemático, dispuesto en dos bandas alargadas a cada lado del xilema. Una disposición similar de los tejidos se observó en las especies de los otros grupos y géneros estudiados.

Los cordones vasculares presentan un proceso de reducción en número y diferenciación en tamaño al pasar del rizoma-filopodio al eje pecíolo-raquis (Fig. 3). En las especies del grupo de Polypodium plesiosorum y en los otros taxa estudiados, se distinguen en la base del pecíolo dos CVG, excepto en Pleopeltis fallax (Schltdl. \& Cham.) Mickel \& Beitel, que presenta uno. Los CVP están ausentes en algunas especies del grupo Polypodium vulgare, $P$. munchii Christ y Pleopeltis fallax. A partir de la base del pecíolo y en dirección acrópeta existe una tendencia a la fusión de los CVP entre sí, de los CVP con los CVG y finalmente de los dos CVG, hasta formar un cordón vascular de forma más o menos triangular en la parte distal del raquis (Fig. 3) a excepción del tipo XI donde se conservan los dos CVG y uno de los CVP. A su vez, este cordón vascular cambia gradualmente la configuración del tejido xilemático en dirección acrópeta; al formarse presenta forma de " $X$ " luego pasa sucesivamente a formas tipo "mariposa", "T" o "yunque" y, finalmente al tipo "hongo" (Fig. 2D). La vena primaria de los segmentos de la lámina tiene un cordón vascular como el descrito en la última fase de las fusiones del eje pecíolo-raquis y se encuentra protegida por capas de células esclerosadas en la parte superior e inferior (Figs. 5A, B).

Se reconocen 11 tipos de configuraciones de los cordones vasculares con base en la fusión/división de los mismos (Fig. 3). Tipo I, Pleopeltis fallax. Tipo II, Polypodium munchii, también presente en las especies europeas del grupo Polypodium vulgare ( $P$. vulgare y $P$. someyae Yatabe) según Rödl-Linder (1990). Tipo IIIa, Polypodium californicum Kaulf. y Synammia feuillei. Tipo IIIb, Polypodium eatonii y P. colpodes. Tipo IIIc, Polypodium polypodioides y Pleopeltis angusta Humb. \& Bonpl. ex Willd. Tipo IV, típico de la mayor parte de las especies de los grupos de Polypodium dulce y $P$. plesiosorum (Polypodium arcanum, $P$. conterminans, $P$. flagellare, $P$. hispidulum, P. martensii, $P$. plesiosorum y $P$. rhodopleuron). Tipo $\mathbf{V}$, especies de del grupo de Polypodium polypodioides (hoja escamosa) con dos variantes: Tipo Va, con fusión de los $\mathrm{CVG}$ en la parte distal del pecíolo (Polypodium alansmithii R.C. Moran, P. fraternum, $P$. guttatum Maxon, $P$. lepidotrichum (Fée) Maxon, $P$. pyrrholepis (Fée) Maxon, P. plebeium Schltdl. \& Cham. y P. remotum Desv.) y Tipo Vb, sin 


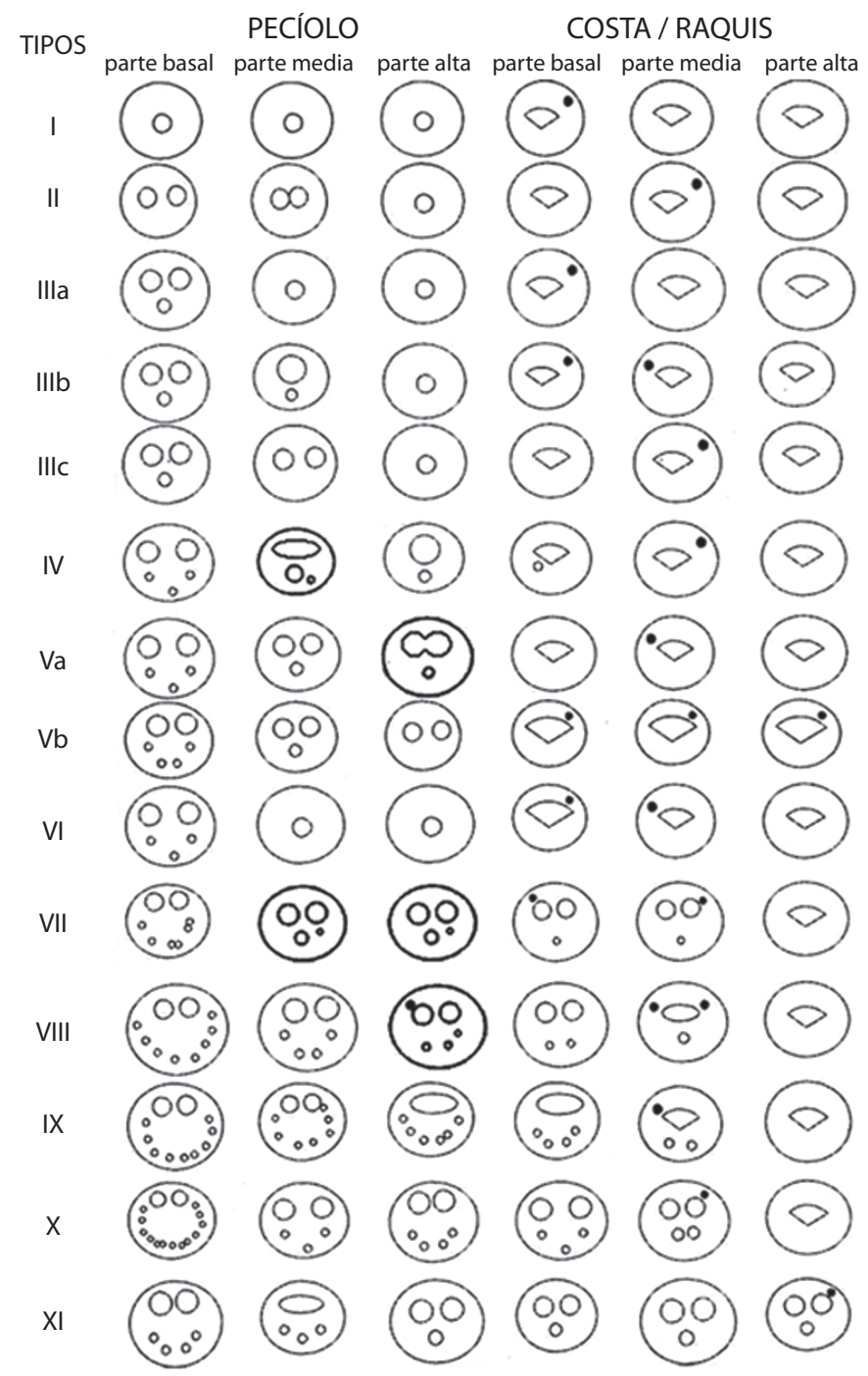

Fig. 3. Patrones de fusión/división de cordones vasculares a lo largo del pecíolo-costa/raquis. Cordones vasculares grandes $(\mathrm{CVG})=$ círculos grandes, cordones vasculares pequeños $(\mathrm{CVP})=$ círculos pequeños y trazas foliares=(•).

Fig. 3. Fusion/fission patterns in vascular strands along petiole-rachis. Large vascular stands (CVG) are represented with large circles, small vascular stands (CVP) with small circles and leaf trace with $(\bullet)$.

fusión de los CVG y desaparición de los CVP en la parte distal del pecíolo (Polypodium furfuraceum y Pleopeltis mexicana (Fée) Mickel \& Beitel). Tipo intermedio entre los tipos IV y V, Polypodium rhachipterygium. Tipo VI, Pecluma dispersa. Tipo VII, Polypodium echinolepis Fée y P. subpetiolatum Hook. Tipo
VIII, Phlebodium pseudoaureum y Polypodium pleurosorum. Tipo IX, Serpocaulon triseriale, Polypodium kunzeanum. Tipo $\mathbf{X}$, definido por Rödl-Linder (1990) quien lo observó en Goniophlebium percussum (Cav.) Wagner \& Grether y G. demersum (Brause) Rodl-Linder. Tipo XI, Serpocaulon falcaria (Kunze) A. R. Sm. 


\section{Lámina}

En vista superficial, las células epidérmicas típicas de ambas superficies tienen tamaños variables y muestran una gradación desde paredes fuertemente lobuladas (Polypodium rhachipterygium) hasta sinuosas (Polypodium guttatum y Phlebodium pseudoaureum). En la superficie abaxial se observan estomas generalmente distribuidos y orientados en dirección al eje longitudinal de la hoja. Se reconocieron cinco tipos estomáticos: anomocítico, copolocítico en dos versiones: copolocítico " $a$ " donde la célula anexa está conectada con dos células opuestas y copolocítico "b" donde la célula anexa está conectada con una célula opuesta; diacítico y polocítico (Figs. 4A-H). En cada una de las 21 especies estudiadas para este carácter, se observó al menos tres de estos tipos en porcentajes variables, con excepción de Synammia feuillei que presentó exclusivamente estomas de tipo polocítico (Cuadro 3). Los tipos anomocítico, copolocítico b y polocítico se presentan en mayor proporción en las especies revisadas (Cuadro 3).

En la epidermis adaxial, cerca de la parte marginal de la lámina, las células epidérmicas están interrumpidas por los hidátodos. Éstos están en conexión con las últimas ramificaciones de las venas foliares y se aprecian, en vista superficial, como una glándula de forma ovada, compuesta de células cuadrangulares dispuestas en círculos concéntricos, unida a una hilera de células alargadas rectangulares (el pie) que corresponde a la parte terminal de la vena (Fig. 4K).

La mayoría de las especies pubescentes de los grupos de Polypodium plesiosorum y $P$. dulce estudiados presentan tricomas cateniformes, hialinos, con una a cuatro células de longitud (Fig. 4J), con excepción de P. echinolepis cuyos tricomas son uni a bicelulares capitados (Fig. 4I).

En vista transversal, las epidermis adaxial y abaxial son de un estrato celular, presentan una cutícula muy delgada y células con forma de domos cortos (Fig. 5A).

En las especies estudiadas se observaron dos tipos de mesofilo. El unifacial, que está formado por células parenquimáticas dispuestas en forma de columna, "Y" o "X" (Fig. 5A), con cámaras subestomáticas rodeadas por células parenquimáticas globosas en la parte abaxial; lo presentaron Polypodium arcanum, P. californicum, P. colpodes, P. hispidulum, P. plesiosorum, P. rhachipterygium, P. rhodopleuron, Phlebodium pseudoaureum y Serpocaulon falcaria. En el mesofilo bifacial (dorsiventral) (Fig. 5B), se observan dos a cuatro capas de células isodiamétricas a cuadrangulares de parénquima denso (en empalizada) hacia la superficie adaxial y un parénquima laxo (esponjoso) hacia la abaxial; lo presentaron Polypodium echinolepis, P. fraternum, P. subpetiolatum, Serpocaulon triseriale y las especies del grupo Polypodium polypodioides y Pleopeltis.

En la zona de la vena primaria se distinguen por debajo de la epidermis varias capas de células con paredes engrosadas que pueden o no estar esclerosadas. La vena primaria presenta una estructura semejante a la descrita para la porción distal del raquis (Fig. 5).

\section{DISCUSIÓN}

Los tipos de anastomosis de las venas secundarias y superiores y la forma en que se fusionan los cordones vasculares a lo largo del eje pecíolo-raquis de la hoja, fueron los caracteres más relevantes para reconocer dos grupos de especies homogéneas en el complejo de Polypodium plesiosorum. Otros caracteres como la división de la lámina, los tipos estomáticos o la anatomía del mesofilo laminar ayudaron a definir similitudes con otros grupos fuera del complejo bajo estudio.

\section{Arquitectura foliar}

\section{Forma y división de la lámina.}

Entre las especies estudiadas del grupo de Polypodium plesiosorum (sensu Moran 1995), la división de la lámina resultó muy variable al encontrarse láminas pinnatisectas (7 especies), pinnatipartidas (1) y pinnadas (3). Los estados intermedios basipinnados que se presentan en 

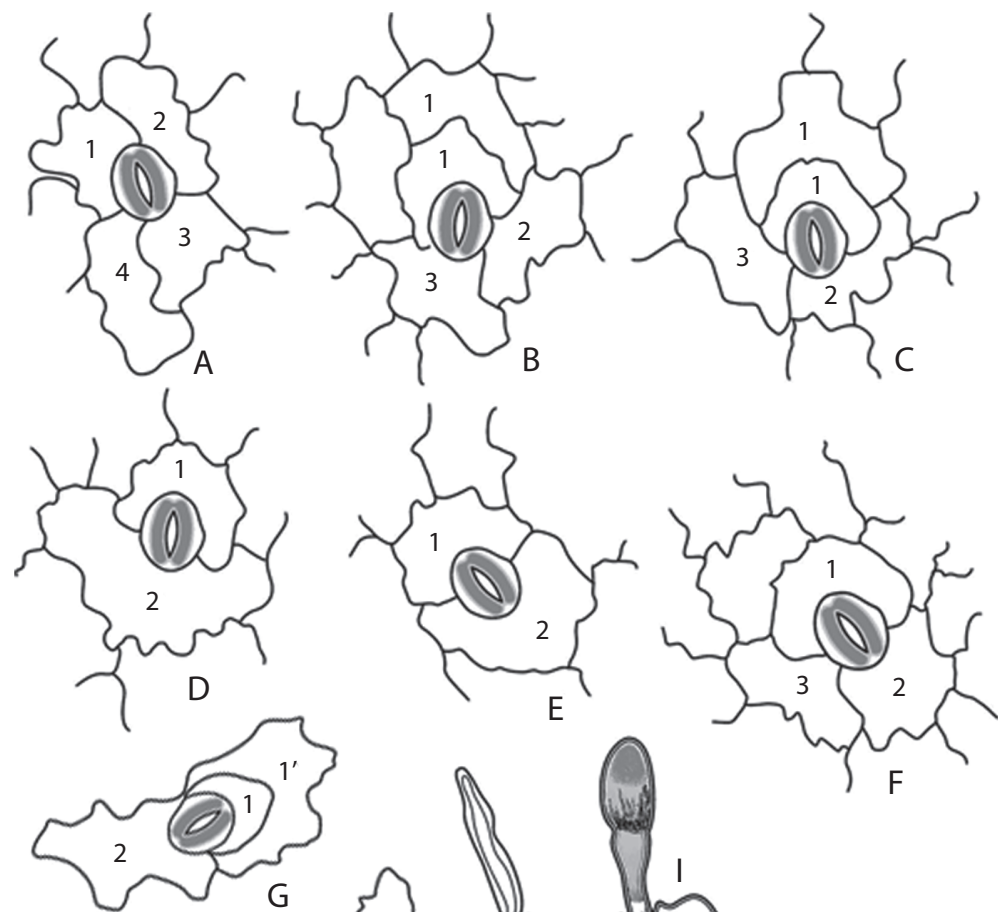

E
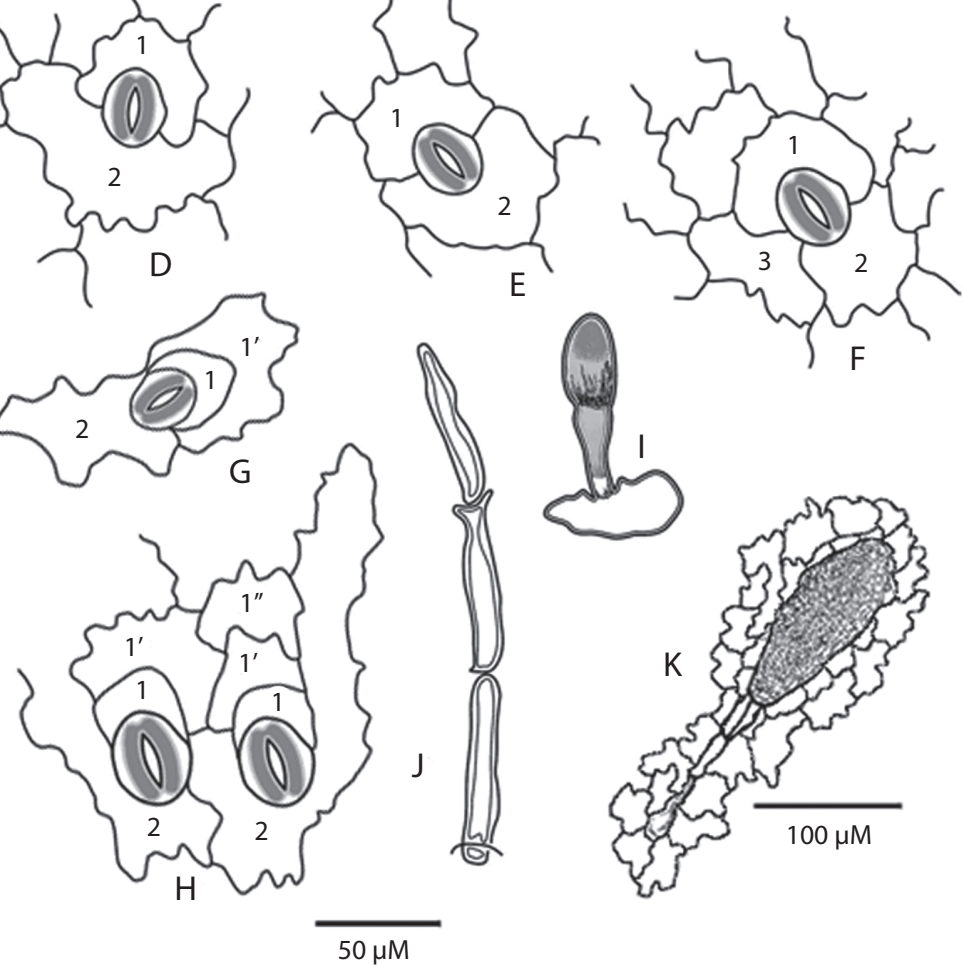

Fig. 4. Estructura epidérmica. Tipos estomáticos $\mathrm{A}-\mathrm{H}$. $\mathrm{A}=$ anomocítico. $\mathrm{B}, \mathrm{F}=$ polocítico. $\mathrm{C}=$ copolocítico. $\mathrm{D}, \mathrm{E}=$ diacítico. $\mathrm{G}$, $\mathrm{H}=$ copolocíticos [G es la variante donde existen una o dos células anexas opuestas y $\mathrm{H}$ es la variante donde existen hasta tres célula anexas opuestas ( $\left.1^{\prime}\right)$. Tricomas I-J. I=tricoma glandular en P. echinolepis. J=tricoma cateniforme común en las especies estudiadas. Hidatodo K.

Los números corresponden a la posición de las células subsidiarias que rodean a las de guarda.

Fig. 4. Epidermal structure. Stomatal types A-H. A=anomocytic. B, F=polocytic. $\mathrm{C}=$ copolocytic. D, E=diacytic. G, $\mathrm{H}=$ copolocytics $[\mathrm{G}$ is a variant where two subsidiary cells are opposited y $\mathrm{H}$ is a variant where three subsidiary cells are opposited. Trichomes I-J. I=gland trichome in P. echinolepis. $\mathrm{J}=$ catenate trichome, common in the studied species. Hydathode K.

Numbers correspond to subsidiary cells position that surround guard ones. 
CUADRO 3

Porcentaje de tipos de aparatos estomáticos en especies del grupo Polypodium plesiosorum y otros taxones estudiados

TABLE 3

Percentage of stomatal apparatus types in species of the Polypodium plesiosorum group and other taxa studied

\begin{tabular}{|c|c|c|c|c|c|}
\hline Taxones & Anomocítico & Polocítico & Diacítico & Copolocítico A & Copolocítico B \\
\hline \multicolumn{6}{|c|}{ Grupo P. plesiosorum } \\
\hline P. arcanum & 16 & 44 & 2 & 0 & 38 \\
\hline P. colpodes & 10 & 48 & 4 & 4 & 34 \\
\hline P. eatonii & 8 & 40 & 10 & 4 & 38 \\
\hline P. flagellare & 2 & 56 & 14 & 0 & 28 \\
\hline P. fraternum & 12 & 54 & 0 & 0 & 34 \\
\hline P. hispidulum & 26 & 42 & 0 & 0 & 26 \\
\hline P. kunzeanum & 6 & 28 & 18 & 4 & 44 \\
\hline P. plesiosorum & 20 & 52 & 0 & 0 & 28 \\
\hline P. pleurosorum & 2 & 26 & 2 & 20 & 50 \\
\hline P. rhachipterygium & 28 & 48 & 2 & 0 & 22 \\
\hline P. rhodopleuron & 4 & 44 & 4 & 0 & 48 \\
\hline \multicolumn{6}{|l|}{ Grupo P. dulce } \\
\hline P. echinolepis & 30 & 58 & 0 & 0 & 12 \\
\hline P. martensii & 16 & 22 & 8 & 12 & 42 \\
\hline \multicolumn{6}{|c|}{ Grupo $P$. polypodioides } \\
\hline P. alan-smithii & 44 & 34 & 6 & 0 & 16 \\
\hline P. guttatum & 50 & 38 & 0 & 0 & 12 \\
\hline P. plebeium & 34 & 46 & 4 & 0 & 16 \\
\hline \multicolumn{6}{|l|}{ Grupo $P$. vulgare } \\
\hline P. californicum & 12 & 38 & 0 & 18 & 32 \\
\hline \multicolumn{6}{|l|}{ PHLEBODIUM } \\
\hline P. pseudoaureum & 28 & 32 & 18 & 2 & 10 \\
\hline \multicolumn{6}{|l|}{ SERPOCAULON } \\
\hline S. falcaria & 4 & 36 & 2 & 8 & 50 \\
\hline S. triseriale & 2 & 34 & 0 & 30 & 34 \\
\hline \multicolumn{6}{|l|}{ SYNAMMIA } \\
\hline S. feuillei & 0 & 100 & 0 & 0 & 0 \\
\hline
\end{tabular}

$P$. castaneum, $P$. colpodes y semipinnada en $P$. flagellare son aparentemente casos derivados de la condición pinnatisecta, dado que sus segmentos basales son adnatos, sursumcurrentes y la porción apical de la lámina es subconforme. En Polypodium eatonii la condición dual del grado de división de la lámina aparentemente es un caso de estrés hídrico (Tejero \& Pacheco 2004). Por otra parte, Polypodium fraternum, P. kunzeanum y P. pleurosorum, son disímiles al resto de las especies del grupo dado que la lámina es pinnada, las pinnas proximales tienen la base cuneada o redondeado-sésil y el segmento apical es conforme o levemente subconforme. En términos generales el contorno de la lámina en las especies del grupo es deltado a ovado lanceolado en diferentes proporciones, dado que no se reducen demasiado los segmentos basales como en Pecluma (ej. P. dispersa). $\mathrm{Si}$ acaso, en algunos ejemplares de Polypodium conterminans, P. hispidulm, P. plesiosorum y $P$. rhodopleuron, la base de la lámina se ve afectada porque los segmentos tienden a estar reflejos. 


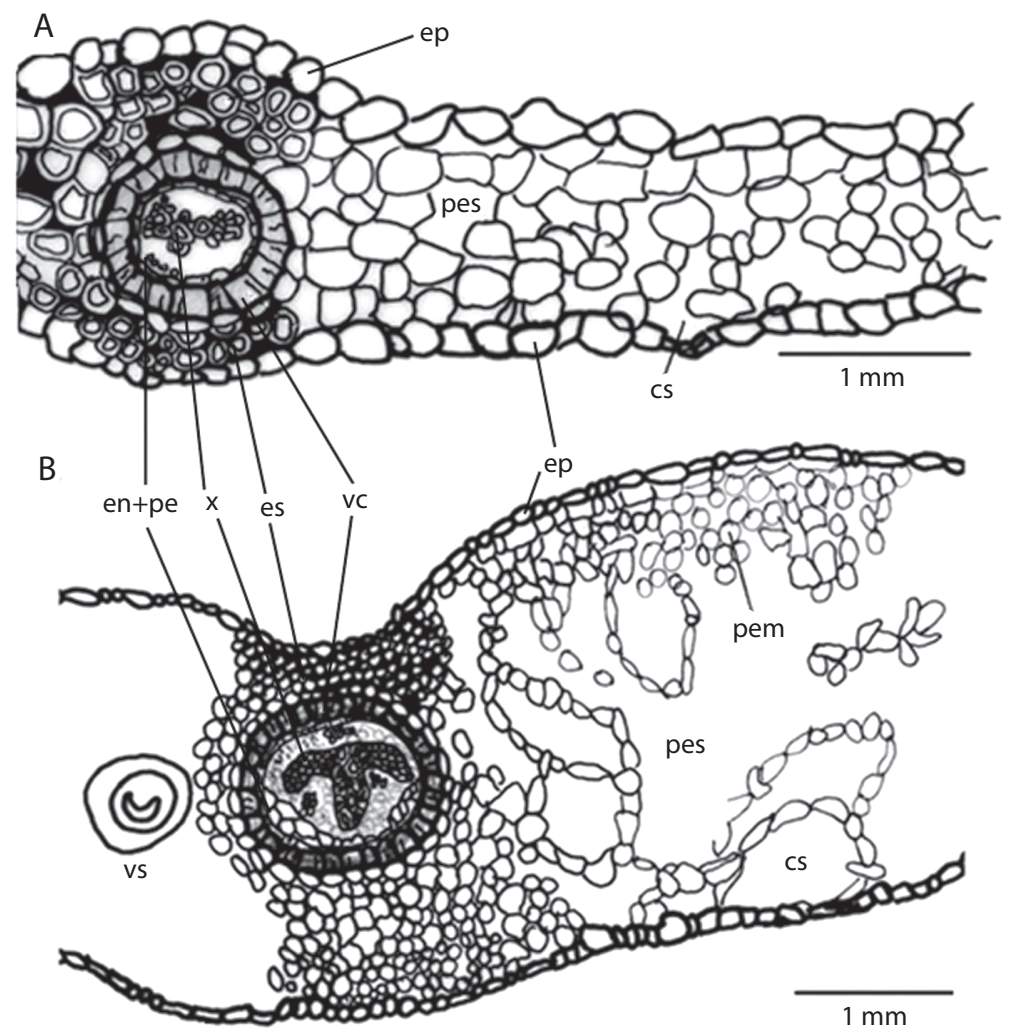

Fig. 5. Corte transversal de lámina en Polypodium. A. Mesofilo unifacial en P. rhodopleuron (Tejero-Díez 4193 IZTA). B. Mesofilo bifacial en $P$. fraternum (D. Tejero-Díez 4245 IZTA).

cs=cámara subestomática; ep=epidermis; en=endodermis; es=esclerénquima; pe=periciclo; pem= parénquima en empalizada; pes=parénquima esponjoso; $\mathrm{vc}=$ vaina circumendodermal; $\mathrm{vs}=$ vena secundaria; $\mathrm{x}=\mathrm{xilema}$.

Fig. 5. Transverse section of blade in Polypodium. A. Unifacial mesophyll in P. rhodopleuron (Tejero-Díez 4193 IZTA). B. Bifacial mesophyll in P. fraternum (D. Tejero-Díez 4245 IZTA).

$\mathrm{cs}=$ substomatal chamber; ep=epidermis en=endodermis; es=sclerenchyma; pe=pericycle; pem=palisade parenchyma; pes=spongy parenchyma; $v c=$ circumendodermal sheath; $v s=$ secondary vein; $x=x y l e m$.

Polypodium rhachipterygium es la única especie del complejo P. plesiosorum con hoja pinnatipartida que además presenta un ala largamente decurrente sobre el pecíolo derivado del par de segmentos proximales; este último carácter lo comparte con varias especies del grupo de $P$. polypodioides, con Pleopeltis y con unas cuantas especies del grupo de Polypodium dulce (sensu Moran 1995).

Polypodium kunzeanum es un caso especial dentro del complejo P. plesiosorum y entre los polipodiaceos de América, ya que además de tener características celulares como la presencia de cristales prismáticos y fibras dispersas en el parénquima del peciolo-raquis, es la única especie con las pinnas articuladas al raquis, carácter que comparte con el género asiático Goniophlebium (Rödl-Linder 1990).

El margen crenulado a crenado es la condición generalizada en las especies estudiadas. Este carácter se puede presentar indistintamente en todo el segmento o sólo en la mitad distal. En ocasiones es poco evidente o bien puede estar muy pronunciado e incluso formar 
lóbulos en el margen acroscópico de los segmentos proximales, como en ciertos individuos de Polypodium plesiosorum var. plesiosorum. La condición de margen crenado aparentemente es común en los polipodios americanos, ya que también aparece en algunas especies del grupo $P$. vulgare, en la mayoría de las especies del grupo $P$. polypodioides y en varias especies del grupo $P$. dulce y de Serpocaulon. Por el contrario, el margen entero a repando entre los polipodiáceos es raro, observándose sólo en Polypodium rhachipterygium y P. eatonii del complejo bajo estudio. Este tipo de margen es propio de los géneros Phlebodium y Pecluma, también se presenta en algunas especies de Pleopeltis, del grupo de Polypodium dulce y Serpocaulon.

\section{Disposición de las venas}

Wagner (1952) y de la Sota (1973) mencionaron que las formas primitivas en los polipodiáceos poseían venas reticuladas y, por tanto, las especies con venas libres, generalmente distribuidas en las zona templadas de ambos hemisferios, pueden considerarse como derivadas. Sin embargo, en el Nuevo Mundo, donde los polipodiáceos han tenido patrones secundarios de evolución (Hennipman et al. 1990, Lellinger 1993), ambos tipos de venas pueden apreciarse; aparentemente en los grupos que se originan en el neotrópico del hemisferio norte surgen venas anastomosadas a partir del complejo de Polypodium vulgare y $P$. dulce de venación abierta (Haufler \& Ranker 1995, Haufler et al. 1995), mientras que los taxa del hemisferio sur las presentan exclusivamente anastomosadas tal como se observa en los géneros Serpocaulon y Synammia.

En los complejos de Polypodium dulce y $P$. vulgare, definidos en parte por su venación abierta, $P$. californicum presenta ocasionalmente aréolas pentagonales; en $P$. echinolepis existen poblaciones con venas anastomosadas $\mathrm{y}$, en $P$. conterminans, generalmente son anastomosadas con proporción de venas libres semejante a $P$. plesiosorum. En el grupo bajo estudio, $P$. fraternum presenta poblaciones con venas libres (Tejero-Diez 4359 IZTA) o anastomosadas (Tejero-Diez 4245 IZTA) y, en menor proporción, también P. rhachipterygium.

Con respecto al tipo de anastomosis de las venas terciarias o de orden superior, las uniones más sencillas aparentemente están representadas por el tipo III. Polypodium conterminans según Moran (1995) y Mickel \& Smith (2004) pertenece al grupo de $P$. dulce; sin embargo, el ejemplar tipo de $P$. conterminans y $80 \%$ de los ejemplares estudiados presentan aréolas pentagonales semejantes a $P$. plesiosorum. Considerando este carácter, junto con la similitud de las escamas del rizoma (Tejero-Díez \& Pacheco en prep.), se propone que $P$. conterminans forme parte del grupo de P. plesiosorum (sensu stricto).

Uniones de venas más complejas están representadas por el tipo IV registradas en Polypodium arcanum, P. castaneum, P. colpodes, $P$. eatonii y $P$. flagellare. Este carácter apoya la propuesta de Tejero-Díez \& Pacheco (2004) de definir a estas especies como un grupo homogéneo.

El tipo V (de aréolas poliangulares), es un carácter que exhibieron Polypodium pleurosorum y P. kunzeanum. La venación parecida de P. pleurosorum con Phlebodium y, la tendencia a dividir la vena fértil como en el grupo VI, han sido argumentos para apoyar la reclasificación de $P$. pleurosorum como un miembro de Phlebodium: Ph. inaequale T. Moore (TejeroDíez et al. 2009). Por otra parte, Polypodium kunzeanum, debido a las particularidades histológicas y anatómicas anotadas, tendrá que reconsiderarse taxonómicamente en el futuro.

Los tipos I a V de organización en las venas estériles se encuentran en las distintas especies del grupo de Polypodium polypodioides y Pleopeltis dando la impresión de un continuum evolutivo, carácter que se complementa con la forma peculiar de división de la vena fértil tipo VII. Esta última forma de ramificación de la vena fértil no se observó en ninguna especie del grupo P. plesiosorum. De la Sota (1966) hace un análisis más detallado de los tipos V y VII de aréolas fértiles en el grupo Polypodium squamatum L. e indica su parecido 
con las del género Pleopeltis. Este estudio y otros similares (Windham 1993, Hooper 1994), junto con los de índole molecular (Schneider et al. 2004, Otto 2007) apoyan la idea de reubicar a las especies del grupo de Polypodium polypodioides dentro de Pleopeltis.

\section{Anatomía}

\section{Peciolo-raquis}

Rödl-Linder (1990) describió en su estudio para Goniophlebium cuatro series de disposiciones de cordones vasculares a lo largo del eje pecíolo-raquis. Además de éstas, en este trabajo se describen otras siete, consideradas como "tipos", a partir del análisis correspondiente en algunas especies de Pecluma, Phlebodium, Pleopeltis y Polypodium. En su conjunto, estos tipos aportan información relevante sobre las relaciones taxonómicas y filogenéticas de Polypodiaceae (Tejero-Díez 2005). Polypodium eatonii y P. colpodes presentan el tipo II junto con $P$. californicum perteneciente al grupo de $P$. vulgare, con el cual según Haufler \& Ranker (1995) algunas especies del complejo P. plesiosorum tiene una relación filogenética. El resto de las especies del grupo de $P$. plesiosorum y P. dulce (Moran, 1995) muestran el tipo III, con excepción de $P$. subpetiolatum y P. echinolepis (especies de hoja pinnada) que presentan mayor cantidad de CVP. Ello hace que estas dos últimas especies puedan formar un grupo propio, más relacionadas a Phlebodium que con las propias del grupo de Polypodium dulce, dentro del cual Moran (1995) las reconoce. Las especies estudiadas de Serpocaulon y Polypodium kunzeanum presentan formas de fusión/división que corresponden a algunas de las series reconocidas por Rödl-Linder (1990) para Goniophlebium, como son los taxa americanos actualmente reconocidos como Serpocaulon y el grupo Goniophlebium argutum (Wall. ex Hook.) J. Sm. ex Hook. respectivamente. De acuerdo con de la Sota (1966), el grupo de Polypodium squamatum presenta el tipo IV, el mismo que se observó en $P$. guttatum y $P$. plebeium (grupo P. pycnocarpum de Tryon \&
Tryon 1982); grupos que Windham (1993) y Otto (2007) consideran como Pleopeltis. Por otra parte el tipo VI observado en Pecluma, está relacionado con el peciolo terete, carácter que es distintivo de este género (Price 1983).

\section{Lámina}

Sen \& Hennipman (1981) reconocieron en la familia Polypodiaceae un total de nueve tipos estomáticos y más de tres tipos se pueden encontrar con diferente predominio en una misma especie. A partir del análisis llevado a cabo, se observó que Synammia feuillei fue la única especie que presentó un único tipo estomático, el polocítico; en el resto de las especies existe combinación de tipos estomáticos semejante a lo citado por Sen \& Hennipman (1981) y ninguno de ellos permite caracterizar algún grupo taxonómico de los aquí considerados. De acuerdo con Crabbe et al. (1975) y PichiSermolli (1977), en Polypodium la presencia del tipo polocítico aunado a los tipos copolocítico y anomocítico es una condición primitiva, característica de Polypodiaceae (incluyendo a los helechos grammitidáceos, Smith et al. 2006). En este sentido, llama la atención la presencia de un sólo tipo estomático en Synammia feuillei; especie de distribución austral que según Schneider et al. (2006) constituye una línea filogenética independiente de Polypodiaceae en Sudamérica, lejanamente relacionada con el grupo Serpocaulon tal como había sugerido Tryon \& Tryon (1982).

Con respecto al mesofilo, los grupos Polypodium plesiosorum y P. vulgare presentan una lámina con estructura unifacial, con excepción de $P$. fraternum que es bifacial, mientras que las especies estudiadas de los grupos $P$. dulce y $P$. polypodioides y en Pleopeltis y Serpocaulon la tienen bifacial. Aparentemente, entre los helechos polipodiáceos la condición bifacial podría ser una situación derivada, adquirida como consecuencia de la adaptación a medios ecológicos xerófilos, pero es interesante señalar que esta condición de la lámina es el común denominador en los grupos y géneros señalados. 
Finalmente, la presencia de aréolas pentagonales y la forma y tipo de división de la lámina, permitieron agrupar a Polypodium conterminans (originalmente considerado en el grupo de $P$. dulce de venación libre sensu Moran 1995) con P. hispidulum, P. plesiosorum y P. rhodopleuron. Estas cuatro especies comparten caracteres similares, además de las mencionadas, en las escamas del rizoma y por ello pueden constituir el grupo P. plesiosorum típico.

Con base en la forma en que se anastomosan las venas (tipo IV) y a la distribución de los cordones vasculares a lo largo del eje pecíolo-raquis, este trabajo apoya la propuesta de Tejero-Díez \& Pacheco (2004) con relación a que Polypodium arcanum, P. castaneum, P. colpodes, $P$. eatonii y $P$. fagellare forman un grupo homogéneo, denominado grupo $P$. colpodes.

Polypodium fraternum, $P$. kunzeanum, $P$. pleurosorum y $P$. rhachipterygium no tienen el patrón de caracteres de los grupos de $P$. plesiosorum o P. colpodes descritos. Polypodium fraternum por su hoja pinnada, venación tipo I y III, cordones vasculares Tipo V, aunado a la presencia de escamas comosas en el rizoma (Tejero-Díez 2005), es posible que pertenezca a una sección cercana al grupo de Polypodium dulce de Moran (1995). La especie sudamericana Polypodium kunzeanum presenta las pinnas articuladas al raquis, carácter único en las especies de la familia Polypodiaceae en América. El carácter anterior y el patrón de fusión y división de cordones vasculares en el pecíolo-raquis tipo IX, la hace semejante a Goniophlebium propio de Asia. Polypodium pleurosorum, por su sistema de venas anastomosado tipo $\mathrm{V}$ y sistema de cordones vasculares del pecíolo-raquis tipo VIII, se relaciona con el género Phlebodium; por ello Tejero-Díez et al. (2009) sugieren volver a utilizar el nombre Phlebodium inaequale T. Moore que Moore (1855) le asignó. Polypodium rhachipterygium, que presenta venación tipo I y III, lámina pinnatipartida y ala peciolar, aunado a caracteres propios del rizoma y soro (Tejero-Díez 2005), permite vislumbrar su relación con el grupo de P. dulce.

\section{AGRADECIMIENTOS}

El presente estudio se realizó gracias a la beca de doctorado 160253 otorgada al primer autor por el Consejo Nacional de Ciencia y Tecnología. Las laminillas histológicas fueron realizadas por Alexander Rojas Alvarado, bajo la supervisión de Estela Sandoval Zapotitla, en el laboratorio de histología vegetal del Jardín Botánico del Instituto de Biología de la UNAM. A Magali Santillán se deben los dibujos y a Alin Torres Díaz la configuración de las figuras. La crítica al manuscrito original (tesis) la realizaron Fernando Chiang Cabrera del Instituto Biología de la UNAM, Adolfo Espejo Serna y Blanca Pérez García del Depto. de Biología de la UAM-Iztapalapa y Francisco Lorea Hernández del Instituto de Ecología A. C. y, para esta versión, colaboraron Blanca Pérez García y Mónica Ponce del Instituto de Botánica Darwinion, Argentina. Los comentarios de cinco dictaminadores anónimos ayudaron a mejorar sustancialmente este manuscrito.

\section{RESUMEN}

El complejo Polypodium plesisorum es un grupo de morfología heterogénea. Se estudió la arquitectura y anatomía foliar de 12 especies que constituyen el complejo de Polypodium plesiosorum reconocidas por Moran, y otras 23 especies de Polypodium (complejos $P$. dulce, $P$. polypodioides y P. vulgare sunsu Moran) у géneros relacionados taxonómicamente (Goniophlebium, Phlebodium, Pleopeltis, Serpocaulon, Synammia) con fines comparativos. Dos grupos de especies homogéneas fueron establecidos en el complejo P. plesisorum. Uno de ellos se reconoce por el tipo de anastomosis de las venas (tipo III): Polypodium conterminans (originalmente considerado en el grupo de $P$. dulce de venación libre según Moran), $P$. hispidulum, $P$. plesiosorum y $P$. rhodopleuron. El segundo grupo formado por Polypodium arcanum, P. castaneum, $P$. colpodes, $P$. eatonii y $P$. flagellare, se reconoce por el tipo de anastomosis de las venas (tipo IV) y la forma en que se fusionan los cordones vasculares a lo largo del eje pecíoloraquis de la hoja. Los caracteres estudiados ayudaron a relacionar las especies restantes con otros grupos fuera del complejo estudiado.

Palabras clave: Polypodiaceae, complejo Polypodium plesiosorum, arquitectura foliar, anatomía foliar, pecíolo. 


\section{REFERENCIAS}

Aguilar Rodríguez, S. 1998. Apéndice I: Técnicas de laboratorio para el estudio de los embriófitos, p. 247272. In D. Tejero-Díez \& M.P. Granillo-Velázquez (eds.). Plantae; Introducción al estudio de las plantas con embrión. Universidad Nacional Autónoma de México-Facultad de Estudios Superiores Iztacala, Tlalnepantla, Edo. México, México.

Anthony, N.C. \& E.A.C.L.E. Schlepe. 1985. xPleopodiuma putative intergeneric fern hybrid from Africa. Bothalia 15: 555-559.

Beck, C.B., R. Schmid \& G.W. Rothwell. 1982. Stelar morphology and the primary vascular systems of seed plants. Bot. Rev. 48: 691-815.

Bosman, M.T.M. 1991. A monograph of the fern genus Microsorium. Leiden Bot. Ser. 14: 1-161.

Copeland, E.B. 1947. Genera filicum; the genera of ferns. Chronica Botanica. Waltham, Massachusetts, EEUU.

Crabbe, J.A., A.C. Jermy \& J.T. Mickel. 1975. A new generic sequence for pteridophyte herbarium. Fern Gaz. 11: 141-162.

Ching, R.C. 1933. The study of Chinese ferns. XI. Polypodium. Contr. Inst. Bot. Acad. Peiping 2: 31-52.

Ching, R.C. 1940. On natural classification of the family "Polypodiaceae". Sunyatsenia 5: 201-268.

Christensen, C. 1928. On the systematic position of Polypodium vulgare. Dansk Bot. Ark. 5: 1-10.

De la Sota, E.R. 1963. Conceptos sobre la evolución de la venación en las filices leptosporangiadas superiores. Ameghiniana 3: 37-42.

De la Sota, E.R. 1965. Las especies escamosas del género Polypodium (s. str.) en Brasil. Rev. Mus. La Plata, Secc. Bot. 9: 243-271.

De la Sota, E.R. 1966. Revisión de las especies americanas del grupo Polypodium squamatum L. Rev. Mus. La Plata, Secc. Bot. 10: 69-186.

De la Sota, E.R. 1973. On the classification and phylogeny of the Polypodiaceae, p. 229-244. In A.C. Jermy, J.A. Crabbe \& B.A. Thomas (eds.). The phylogeny and classification of the ferns. J. Linn. Soc. Bot. 67(1).

Evans, A.M. 1969. Interspecific relationships in the Polypodium pectinatum-plumula complex. Ann. Missouri Bot. Gard. 55: 193-293.
Fryns-Claessens, E. \& W. van Cotthem. 1973. A new classification of the ontogenetic types of stomata. Bot. Rev. 39: 71-137.

Gómez, L.D. 1975. Contribuciones a la pteridología costarricense VIII: La hibridización en el trópico; Microgramma x Polypodium y Polypodium aspidiolepis Baker. Brenesia 6: 49-57.

Haufler, C.H. \& T.A. Ranker. 1995. Rbcl sequences provide phylogenetic insights among sister species of the fern genus Polypodium. Amer. Fern J. 85: 361-374.

Haufler, C.H., D.E. Soltis \& P.S. Soltis. 1995. Phylogeny of the Polypodium vulgare complex: insights from chloroplast DNA restriction site data. Syst. Bot. 20: 110-119.

Hennipman, E.P. 1986. The systematics of the Polypodiaceae (Filicales): posing the problems. Acta Bot. Neerl. 35: 115.

Hennipman, E., P. Veldhoen \& K.U. Kramer. 1990. Polypodiaceae, p. 203-230. In K. Kubitzki (ed.). The families and genera of vascular plants. Springer, Berlín, Alemania.

Hensen, R.V. 1990. Revision of the Polypodium loriceum complex (Filicales, Polypodiaceae). Nova Hedwigia 50: 279-336.

Hetterscheid, W.L.A. \& E.P. Hennipman. 1984. Venation patterns, soral characteristics and shape of the fronds of the microsorioid Polypodiaceae. Bot. Jahrb. Syst. 105: 11-47.

Hooper, E.A. 1994. Biosystematic analysis of the Pleopeltis macrocarpa complex in the Neotropics. Ph.D. Thesis, University of Kansas, Kansas, EEUU.

Iwatsuki, K. 1962. On the reticulate venation of the Thelypteroid ferns. Acta Phytotaxonom. Geobot. 20: 219-227.

Johansen, D.A. 1940. Plant microtechnique. McGraw-Hill, New York, EEUU.

Kaur, S. 1978. Contributions to the morphology of Tectaria: venation pattern of sterile and fertile leaves. Phytomorphology 28: 14-19.

Lellinger, D.B. 1993. Application of the name Goniophlebium and new subgeneric name in Polypodium. Amer. Fern J. 83: 37-38.

Lloyd, R.M. \& F.A. Lang. 1964. The Polypodium vulgare complex in North America. Brit. Fern Gaz. 9: 168177 . 
Lucansky, T.W. \& R.A. White. 1974. Comparative studies of the nodal and vascular anatomy in the neotropical Cyatheaceae. III. Nodal and petiole patterns; summary and conclusions. Amer. J. Bot. 61: 472-480.

Maxon, W.R. 1903. Studies of certain Mexican and Guatemalan species of Polypodium. Contr. U.S. Natl. Herb. 8: $271-280$.

Maxon, W.R. 1916a. Polypodium furfuraceum and related species. Contr. U.S. Natl. Herb. 17: 557-579.

Maxon, W.R. 1916b. Polypodium squamatum and its allies. Contr. U.S. Natl. Herb. 17: 579-596.

Maxon, W.R. 1916c. Studies of tropical American ferns: No 6 Contr. U.S. Natl. Herb. 17: 541-608.

Mickel, J.T. \& J.M. Beitel. 1987. Notes on xPleopodium and Pleopeltis in tropical America. Amer. Fern J. 77: 16-27.

Mickel, J.T. \& J.M. Beitel. 1988. Pteridophyte flora of Oaxaca, Mexico. Mem. New York Bot. Gard. 46: $1-568$.

Mickel, J.T. \& A.R. Smith. 2004. The pteridophytes of Mexico. Mem. New York Bot. Gard. 88: 1-1054.

Mitsuta, S. 1984. Studies in the venation of Polypodiaceae II. Venation of Polypodiaceae (2) and some other ferns. Mem. Fac. Sci. Kyoto Univ. Ser. Biol. 9: $57-85$.

Moore, T. 1855. New garden ferns. No. IV. 8. Phlebodium inaequale. Gard. Chron. 1855: 660.

Moran, R.C. 1995. Polypodium L., p. 349-365. In R.C. Moran \& R. Riba (eds.). Psilotaceae a Salviniceae. Flora Mesoamericana. Vol 1. Universidad Nacional Autónoma de México, Distrito Federal, México; Missouri Botanical Garden y The Natural History Museum.

Neuroth, R. 1996. Biosystematik und Evolution des Polypodium vulgare-Komplexes (Polypodiaceae, Pteridophyta). Dissert. Bot. 256: 1-209.

Otto, E.M. 2007. Untersuchungen zur Phylogenie der epiphytischen Farngattung Pleopeltis. Diplomarbeit. Albrecht-von-Haller-Institut für Pflanzenwissenschaften an der Biologischen Fakultät der Georg-AugustUniversität zu Göttingen, Göttingen, Alemania.

Payne, W.W. 1968. The use of cellulose acetate film for the production of epidermal cast. Ward's Bull. 7: 6-7.

Pichi-Sermolli, R.E.G. 1977. Tentamen Pteridophytorum genera in taxonomicum ordinem redigendi. Webbia 31: 313-512.
Presl, C.B. 1836. Tentamen pteridographiae. T. Haase, Praga, Rep. Checa.

Price, M.G. 1983. Pecluma, a new tropical American fern genus. Amer. Fern J. 73: 109-116.

Ranker, T.A., A.R. Smith, B.S. Parris, J.M. Geiger, C.H. Haufler, S.C.K. Starub \& H. Schneider. 2004. Phylogeny and evolution of grammitid ferns (Grammitidaceae): a case of rampant morphological homoplasy. Taxon 53: 415-428.

Rödl-Linder, G. 1990. A monograph of the fern genus Goniophlebium (Polypodiaceae). Blumea 34: 277 423.

Saiki, Y., M. Matsumoto \& Y. Mitsuda. 1988. Vascular patterns in the petioles of Aspleniaceae. Proc. ISSP 273-278.

Salgado A.E. 1982. Venation pattern in Philippine Tectaroid fern. De la Salle University, Integrated Research Center. Monograph Series Number 3. Manila, Filipinas.

Salino, A., T.E. Almeida, A.R. Smith, A. Navarro-Gómez, H.P. Kreier \& H. Schneider. 2008. A new species of Microgramma (Polypodiaceae) from Brazil and recircumscription of the genus based on phylogenetic evidence. Syst. Bot. 33: 630-635.

Schneider, H., A.R. Smith, R. Cranfill, T.E. Hildebrand, C.H. Haufler \& T.A. Ranker. 2004. Unraveling the phylogeny of polygrammoid ferns (Polypodiaceae \& Grammitidaceae): exploring aspects of the diversification of epiphytic plants. Molec. Phylogenet. Evol. 31: 1041-1063.

Schneider, H., H.P. Kreier, R. Willson \& A.R. Smith. 2006. The Synammia enigma: evidence for a temperate lineage of polygrammoid ferns (Polypodiaceae, Polypodiidae) in southern South America. Syst. Bot. 31: 31-41.

Schuettpelz, E. \& K.M. Pryer. 2007. Fern phylogeny inferred from 400 leptosporangiate species and three plastid genes. Taxon 56: 1037-1050.

Sen, U. \& E. Hennipman. 1981. Structure and ontogeny of stomata on Polypodiaceae. Blumea 27: 175-201.

Smith, A.R. 1981. Pteridophytes, p. 1-130. In D.E. Breedlove (ed.). Flora of Chiapas Vol. 2. California Academy of Sciences, San Francisco, EEUU.

Smith, A.R., H. Schneider, B.S. Parris, C.H. Haufler, T.A. Ranker, J.P. Terrien \& J.M.O. Geiger. 2000. Phylogeny of Grammitidaceae and Polypodiaceae inferred 
from two large data sets: rbcL and morphology. Amer. J. Bot. 87: 97.

Smith, A.R., H.P. Kreier, C.H. Haufler, T.A. Ranker \& H. Schneider. 2006. Serpocaulon, a new genus segregated from Polypodium. Taxon 55: 919-930.

Steeves, T.A. 1988. Phylogenetic and ontogenetic interpretation of the fern vascular system. Proc. ISSP 279-284.

Stewart, W.N. \& G.W. Rothwell. 1993. Paleobotany and the evolution of plants. Cambridge University, Cambridge, Gran Bretaña.

Stolze, R.G. 1981. Ferns and fern allies of Guatemala. Part II: Polypodiaceae. Fieldiana, Bot. n.s. 6: 1-522.

Tejero-Díez, J.D. 2005. Revisión taxonómica del complejo Polypodium plesiosorum Kunze (Polypodiaceae, Polypodiophyta). Tesis Doctorado, Universidad Autónoma Metropolitana, Distrito Federal, México.

Tejero-Díez, J.D. \& L. Pacheco. 2004. Taxa nuevos, nomenclatura, redefiniciones y distribución en las especies relacionadas a Polypodium colpodes Kunze (Polypodiaceae, Pteridophyta). Acta Bot. Mex. 67: 75-115.

Tejero-Díez, J.D., J.T. Mickel \& A.R. Smith. 2009. A hybrid Phlebodium (Polypodiaceae, Polypodiophyta) and its influence on the circumscription of the genus. Amer. Fern J. 99: 109-116.

Tindale, M.D. 1960. Vein patterns in Microsorium scandens and its allies. Amer. Fern J. 50: 241-245.

Tryon, R.M. 1964. Evolution in the leaf of living ferns. Mem. Torrey Bot. Club 21: 73-82.

Tryon, R.M. 1970. The classification of the Cyatheaceae. Contr. Gray Herb. 200: 3-53.
Tryon, R.M. \& A.F. Tryon. 1982. Ferns and allied plants, with special reference to tropical America. Springer, New York, EEUU.

Wagner, W.H. 1952. Types of foliar dichotomy in living ferns. Amer. J. Bot. 39: 578-592.

Wagner, W.H. 1979. Reticulate veins in the systematics of modern ferns. Taxon 28: 87-95.

Wagner, W.H. 1986. The new world fern genus Marginariopsis (Polypodiaceae): an example of leaf dimorphy and coenosory in generic delimitation. Bull. Torrey Bot. Club 113: 159-167.

Wagner, W.H. \& F.S. Wagner. 1975. A hybrid polypody from the new world tropics. Fern Gaz. 11: 125-135.

Wardlaw, C.W. 1944. Experimental and analytical studies of pteridophytes. IV. Stelar morphology: experimental observations on the relation between leaf development and stelar morphology in species of Dryopteris and Onoclea. Ann. Bot. 8: 387-399.

Weatherby, C.A. 1922. The group of Polypodium lanceolatum in North America. Contr. Gray Herb. 65: 3-14.

Weatherby, C.A. 1939. The group of Polypodium polypodioides. Contr. Gray Herb. 124: 22-35.

Weatherby, C.A. 1947. Polypodium lepidopteris and its relatives in Brazil. Contr. Gray Herb. 165: 76-82.

White, R.A. 1984. Comparative development of vascular tissue patterns in the shoot apex of ferns, p. 53-107. In R.A. White y W.C. Dickison (eds.). Contemporary problems in plant anatomy. Academic, New York, EEUU.

Windham, M.D. 1993. New taxa and nomenclatural changes in the North American fern flora. Contr. Univ. Michigan Herb. 19: 31-61. 


\section{APÉNDICE I}

Especímenes de referencia (estudio anatómico). Especies de Polypodium en grupos de acuerdo con Moran (1995) excepto Serpocaulon (=P. loriceum). Recolectas de D. Tejero-Díez. Unidades morfológicas de estudio: 1=histología del pecíolo y lámina. $2=$ aparato estomático. $3=$ series pecíolo raquis. $4=$ arquitectura foliar

\section{APPENDIX I}

Vouchers used for anatomy study. Polypodium species are arranged in groups agreement with Moran (1995) except Serpocaulon (=P. loriceum). Collect of D. Tejero-Díez. Morphologic units of study: $1=$ histology of petiole and blade. $2=$ stomatal apparatus. $3=$ petiole rachis series. $4=$ leaf architecture

\begin{tabular}{|c|c|c|c|c|c|}
\hline \multirow{2}{*}{$\begin{array}{l}\text { Taxones } \\
\text { POLYPODIUM }\end{array}$} & \multirow[t]{2}{*}{ Referencia } & \multicolumn{4}{|c|}{$\begin{array}{l}\text { Unidades } \\
\text { morfológicas de } \\
\text { estudio }\end{array}$} \\
\hline & & 1 & 2 & 3 & 4 \\
\hline \multicolumn{6}{|l|}{ Grupo P. plesiosorum } \\
\hline \multirow[t]{2}{*}{ P. arcanum var. arcanum } & $\begin{array}{l}4218 \text { (IZTA, UAMIZ); Méx., Pue., Zacapoaxtla, ca. Zacapoaxtla } \\
4230 \text { (IZTA, UAMIZ); Méx., Hgo., km } 22 \text { entre Metepec y Tenango } \\
\text { de Doria }\end{array}$ & $\mathrm{X}$ & $\mathrm{X}$ & $\mathrm{X}$ & \\
\hline & $\begin{array}{l}4240 \text { (IZTA, UAMIZ); Méx., Hgo., Agua Blanca, salto San Pedrito } \\
4250 \text { (IZTA, UAMIZ); Méx., Hgo., Molango, Laguna Atezca } \\
4269 \text { (IZTA, UAMIZ); Méx., Oax., Ixtlán de Juárez, km } 142 \\
\text {-carretera fed. } 175\end{array}$ & & $\mathrm{X}$ & $\mathrm{X}$ & $\begin{array}{l}X \\
X \\
X\end{array}$ \\
\hline $\begin{array}{l}\text { P. arcanum var. septentrionale } \\
\text { Mickel }\end{array}$ & $\begin{array}{l}4160 \text { (IZTA, UAMIZ); Méx., Tamps., Ocampo (km } 30 \text { entre } \\
\text { Ocampo y Tula) }\end{array}$ & $\mathrm{X}$ & $\mathrm{X}$ & $\mathrm{X}$ & $\mathrm{X}$ \\
\hline $\begin{array}{l}\text { P. arcanum var. bakeri Tejero } \\
\text { \& Mickel }\end{array}$ & $\begin{array}{l}4364 \text { (IZTA, UAMIZ); Méx., Gro., Heliodoro Castillo, ca. El } \\
\text { Carrizal }\end{array}$ & & & & $\mathrm{X}$ \\
\hline & 4365 (IZTA, UAMIZ); ibídem, entre Filo de Caballo y Pto. El Gallo & & & & $\mathrm{X}$ \\
\hline P. castaneum & $\begin{array}{l}\text { T. B. Croat } 42213 \text { (MO); El Salvador, Sonsonate, ca. cúspide Cerro } \\
\text { Verde }\end{array}$ & $\mathrm{X}$ & $\mathrm{X}$ & $\mathrm{X}$ & $\mathrm{X}$ \\
\hline \multirow[t]{5}{*}{ P. colpodes var. colpodes } & $\begin{array}{l}2074 \text { (IZTA); Méx., Méx., Almoloya de Alquisiras, entronque a } \\
\text { Ixtapan Los Sabinos } \\
3194 \text { (IZTA); Méx., Méx., Sto. Tomás de los Plátanos, base de la } \\
\text { presa }\end{array}$ & $\mathrm{X}$ & & & \\
\hline & $\begin{array}{l}\text { 4302-4303 (CR, IZTA, UAMIZ); Costa Rica, Guanacaste, P. Nal. } \\
\text { Palo Verde }\end{array}$ & & & & $\mathrm{X}$ \\
\hline & $\begin{array}{l}4313 \text { (CR, IZTA, UAMIZ); Costa Rica, San José, Dota, entre Sta. } \\
\text { María y COPEI }\end{array}$ & & $\mathrm{X}$ & $\mathrm{X}$ & \\
\hline & 4360 (IZTA, UAMIZ); Méx., Chis., Ixtapa, Pte. Río Lajas & & & & $\mathrm{X}$ \\
\hline & $\begin{array}{l}4368 \text { (IZTA); Méx., Gro., Eleodoro Castillo, camino a Puerto El } \\
\text { Gallo }\end{array}$ & & & & $\mathrm{X}$ \\
\hline $\begin{array}{l}\text { P. colpodes var. oligomeron } \\
\text { (H. Christ)Tejero }\end{array}$ & $\begin{array}{l}4311 \text { (CR, INB, IZTA, UAMIZ); Costa Rica, San José, Dota, ca. } \\
\text { Copei. }\end{array}$ & $\mathrm{X}$ & & & \\
\hline P. eatonii & $\begin{array}{l}4315 \text { (IZTA, UAMIZ); Méx., Ver., Banderilla, a } 3 \mathrm{~km} \text { al E de } \\
\text { Banderilla }\end{array}$ & $\mathrm{X}$ & $\mathrm{X}$ & $\mathrm{X}$ & $\mathrm{X}$ \\
\hline P. flagellare & $\begin{array}{l}4306 \text { (CR, INB, IZTA, UAMIZ); Costa Rica, Puntarenas, Garabito, } \\
\text { P. Nal. Carará }\end{array}$ & & $X$ & $\mathrm{X}$ & \\
\hline
\end{tabular}




\begin{tabular}{|c|c|c|c|c|c|}
\hline \multirow[t]{2}{*}{ Taxones } & \multirow{2}{*}{$\begin{array}{l}\text { Referencia } \\
4314 \text { (CR, INB, IZTA, UAMIZ); Costa Rica, San José, Puriscal, ca. } \\
\text { San Martín }\end{array}$} & \multicolumn{4}{|c|}{$\begin{array}{l}\text { Unidades } \\
\text { morfológicas de } \\
\text { estudio }\end{array}$} \\
\hline & & $\mathrm{X}$ & & & $\mathrm{X}$ \\
\hline & $\begin{array}{l}\text { C. W. Dodge } 16567 \text { (MO); Panamá, Chiriquí, Alajuela, ca. Madden } \\
\text { Daan }\end{array}$ & $\mathrm{X}$ & & & \\
\hline & L. D. Gómez 467 (CR); Costa Rica, San Pablo Turrubares & $\mathrm{X}$ & & & \\
\hline \multirow[t]{2}{*}{ P. fraternum } & $\begin{array}{l}4202 \text { (IZTA, UAMIZ); Méx., Pue., Zacapoaxtla, Apulco, salto La } \\
\text { Gloria }\end{array}$ & $\mathrm{X}$ & $\mathrm{X}$ & $\mathrm{X}$ & $\mathrm{X}$ \\
\hline & 4245 (IZTA, UAMIZ); Méx., Hgo., Zacualtipán, ca. Carpinteros & $\mathrm{X}$ & $\mathrm{X}$ & $\mathrm{X}$ & $\mathrm{X}$ \\
\hline \multirow[t]{4}{*}{ P. hispidulum } & $\begin{array}{l}\text { E. Martínez \& G. Aguilar } 10007 \text { (MEXU); Méx., Chis., Ocosingo, } \\
\text { ca. Bonampak }\end{array}$ & $\mathrm{X}$ & & & \\
\hline & H. Türckheim 8053 (P); Guatemala, Alta Verapaz, Cubilguitz & $\mathrm{X}$ & & & \\
\hline & 4328 (IZTA, UAMIZ); Méx., Chis., Palenque, zona arqueológica & & $\mathrm{X}$ & $\mathrm{X}$ & \\
\hline & 4329 (IZTA, UAMIZ); Méx., Chis., salto Misol-Ha & & & & $\mathrm{X}$ \\
\hline \multirow[t]{3}{*}{ P. kunzeanum } & M. Grayum 8602 (INB); Costa Rica, San José, Puriscal, Cerro Bola & $\mathrm{X}$ & & & \\
\hline & C. Linderman 2599 (BM); Brazil, Matto Grosso, cascada Cupin & $\mathrm{X}$ & & & \\
\hline & H. H. Smith 2564 (L, U); Colombia, sin loc. & & $\mathrm{X}$ & & $\mathrm{X}$ \\
\hline \multirow[t]{6}{*}{$\begin{array}{l}\text { P. plesiosorum var. } \\
\text { plesiosorum }\end{array}$} & $\begin{array}{l}4129 \text { (IZTA, UAMIZ); Méx., Qro., Pinal de Amoles, entre } \\
\text { Cadereyta y Pinal A. }\end{array}$ & $\mathrm{X}$ & $\mathrm{X}$ & $\mathrm{X}$ & \\
\hline & $\begin{array}{l}4132 \text { (IZTA, UAMIZ); Méx., Qro., Pinal de Amoles, entre Pinal } \\
\text { Amoles. y Jalpan }\end{array}$ & & $\mathrm{X}$ & $\mathrm{X}$ & \\
\hline & $\begin{array}{l}\text { 4137-4138 (IZTA, UAMIZ); Méx., Tamps., Gómez Farías, reserva } \\
\text { de la biosfera. El Cielo. }\end{array}$ & & $\mathrm{X}$ & $X$ & \\
\hline & $\begin{array}{l}\text { 4205-4206 (IZTA, UAMIZ); Méx., Pue., Zacapoaxtla, Apulco, salto } \\
\text { La Gloria }\end{array}$ & & $\mathrm{X}$ & $\mathrm{X}$ & $\mathrm{X}$ \\
\hline & 4220 (IZTA, UAMIZ); Méx., Pue., Zacapoaxtla, ca. Zacapoaxtla & $\mathrm{X}$ & & & \\
\hline & $\begin{array}{l}4330 \text { (IZTA, UAMIZ); Méx., Ver., Maltrata, km 235-carretera fed. } \\
150\end{array}$ & & & & $\mathrm{X}$ \\
\hline $\begin{array}{l}\text { P. plesiosorum var. } \\
\text { rubicundum Tejero }\end{array}$ & 4308 (INB, IZTA, UAMIZ); Costa Rica, Cartago, Paraíso, ca. Orosí & $\mathrm{X}$ & $\mathrm{X}$ & $\mathrm{X}$ & $\mathrm{X}$ \\
\hline \multirow[t]{2}{*}{ P. pleurosorum } & $\begin{array}{l}4366 \text { (IZTA, UAMIZ); Méx., Gro., Heliodoro Castillo, ca. Filo de } \\
\text { Caballo }\end{array}$ & & & $\mathrm{X}$ & $\mathrm{X}$ \\
\hline & $\begin{array}{l}\text { M. Pérez Farrera } 246 \text { (UAMIZ); Méx., Chis, Villaflores, cerro Tres } \\
\text { Picos }\end{array}$ & & $\mathrm{X}$ & & \\
\hline \multirow[t]{3}{*}{ P. rhachipterygium } & 4185 (IZTA, UAMIZ); Méx., Ver., Catemaco, ca. Coyame & $\mathrm{X}$ & $\mathrm{X}$ & $\mathrm{X}$ & $\mathrm{X}$ \\
\hline & $\begin{array}{l}\text { Ramamoorthy } 3348 \text { (IZTA, MEXU); Méx., Ver., San Andrés } \\
\text { Tuxtla, estación biológca UNAM }\end{array}$ & $\mathrm{X}$ & & & $\mathrm{X}$ \\
\hline & Ramamoorthy 4157 (IZTA, MEXU); Ibídem & & & & $\mathrm{X}$ \\
\hline \multirow[t]{4}{*}{ P. rhodopleuron } & $\begin{array}{l}4078 \text { (IZTA, UAMIZ) ; Méx., Hgo., Tlalchinol, entrada a Lontla } 5 \\
\text { km a Huejutla }\end{array}$ & $\mathrm{X}$ & $\mathrm{X}$ & $\mathrm{X}$ & \\
\hline & $\begin{array}{l}4193 \text { (IZTA, UAMIZ); Méx., Pue., Zacapoaxtla, Apulco, salto La } \\
\text { Gloria }\end{array}$ & $\mathrm{X}$ & $\mathrm{X}$ & & $\mathrm{X}$ \\
\hline & $\begin{array}{l}4228 \text { (IZTA, UAMIZ) ; Méx., Hgo., Tenango de Doria, km } 19 \text { carr. } \\
\text { estatal } 126\end{array}$ & & $\mathrm{X}$ & $\mathrm{X}$ & \\
\hline & 4243 (IZTA, UAMIZ); Méx., Hgo., Zacualtipán, ca. Carpinteros & & $\mathrm{X}$ & & \\
\hline \multicolumn{6}{|l|}{ Grupo $P$. dulce } \\
\hline P. conterminans & 4358 (IZTA, UAMIZ); Méx., Chis., Jitotol, ca. Pueblo Nuevo & $\mathrm{X}$ & $\mathrm{X}$ & $\mathrm{X}$ & $\mathrm{X}$ \\
\hline P. echinolepis & 4213 (IZTA); Méx., Pue., Cuetzalán, salto San Andrés & $\mathrm{X}$ & $\mathrm{X}$ & $\mathrm{X}$ & $\mathrm{X}$ \\
\hline P. martensii & 4088 (ENCB, IZTA), Méx., Hgo., Zacualtipán, ca. Zoquizoquiapán & & $\mathrm{X}$ & $X$ & $X$ \\
\hline
\end{tabular}




\begin{tabular}{|c|c|c|c|c|c|}
\hline Taxones & Referencia & & & & \\
\hline P. subpetiolatum & $\begin{array}{l}4180 \text { (IZTA, UAMIZ); Méx., Méx., Chapa de Mota, ca. Chapa de } \\
\text { Mota. }\end{array}$ & $\mathrm{X}$ & $\mathrm{X}$ & $\mathrm{X}$ & \\
\hline & 4363 (IZTA, UAMIZ); Méx., Chis., P. Nal. Cañón Sumidero & & & & $\mathrm{X}$ \\
\hline Grupo P. polypodioides & & & & & \\
\hline P. alan-smithii & 4074 (IZTA); Méx., Hgo., Tlalchinol, ca. Zacualtipán & $\mathrm{X}$ & $\mathrm{X}$ & $\mathrm{X}$ & $\mathrm{X}$ \\
\hline P. furfuraceum & 4355 (IZTA, UAMIZ); Méx., Oax., Ixtlán de Juárez, ca. Calpulalpan & & & $\mathrm{X}$ & $\mathrm{X}$ \\
\hline P. guttatum & $\begin{array}{l}4192 \text { (IZTA, UAMIZ); Méx., Pue, Acatzingo, km } 27 \text { rumbo a San } \\
\text { Salvador. El Seco }\end{array}$ & $\mathrm{X}$ & $\mathrm{X}$ & $\mathrm{X}$ & $\mathrm{X}$ \\
\hline P. lepidotrichum & $\begin{array}{l}4332 \text { (IZTA, UAMIZ); Méx., Hgo, Zacualtipán, a } 2 \text { km desv. a } \\
\text { Tianguistengo }\end{array}$ & & & $\mathrm{X}$ & $\mathrm{X}$ \\
\hline & $\begin{array}{l}4340 \text { (IZTA, UAMIZ); Méx., Pue., Zacapoaxtla, Apulco, salto La } \\
\text { Gloria }\end{array}$ & & & $\mathrm{X}$ & $\mathrm{X}$ \\
\hline & de la Sota (1966), fig. 12.D & & $\mathrm{X}$ & & \\
\hline P. munchii & 4353 (IZTA, UAMIZ); Méx., Oax., km 110-carretera fed. 175 & & & $\mathrm{X}$ & $\mathrm{X}$ \\
\hline P. plebeium & $\begin{array}{l}4194 \text { (IZTA, UAMIZ); Méx., Pue., Zacapoaxtla, Apulco, salto La } \\
\text { Gloria }\end{array}$ & & $\mathrm{X}$ & $\mathrm{X}$ & $\mathrm{X}$ \\
\hline P. polypodioides & 4346 (IZTA, UAMIZ); Méx., Pue., Cuetzalán, salto San Andrés & & & $\mathrm{X}$ & $\mathrm{X}$ \\
\hline P. pyrrholepis & 4356 (IZTA, UAMIZ); Méx., Oax., Ixtlán de Juárez, ca. Calpulalpan & & & $\mathrm{X}$ & $\mathrm{X}$ \\
\hline P. remotum & $\begin{array}{l}4333 \text { (IZTA, UAMIZ); Méx., Hgo, Zacualtipán, a } 2 \text { km desv. a } \\
\text { Tianguistengo }\end{array}$ & & & & $\mathrm{X}$ \\
\hline Grupo $P$. vulgare & & & & & \\
\hline P. californicum & $\begin{array}{l}\text { Sánchez Glez. } 10 \text { V } 2001 \text { (CHAPA, IZTA); Méx., Méx., Texcoco, } \\
\text { cerro Tláloc }\end{array}$ & $\mathrm{X}$ & $\mathrm{X}$ & $\mathrm{X}$ & $\mathrm{X}$ \\
\hline GONIOPHLEBIUM & & & & & \\
\hline G. benguetense & Rödl-Linder (1990), fig. 3.a & $\mathrm{X}$ & $\mathrm{X}$ & $\mathrm{X}$ & $\mathrm{X}$ \\
\hline PECLUMA & & & & & \\
\hline P. dispersa & $\begin{array}{l}4341 \text { (IZTA, UAMIZ); Méx., Pue., Zacapoaxtla, Apulco, salto La } \\
\text { Gloria }\end{array}$ & & & $\mathrm{X}$ & $\mathrm{X}$ \\
\hline PHLEBODIUM & & & & & \\
\hline P. pseudoaureum & $\begin{array}{l}4195 \text { (IZTA, UAMIZ); Méx., Pue., Zacapoaxtla, Apulco, salto La } \\
\text { Gloria }\end{array}$ & $\mathrm{X}$ & $\mathrm{X}$ & $\mathrm{X}$ & $\mathrm{X}$ \\
\hline Phlebodium xhemipinnatum & 4362 (IZTA, UAMIZ); Méx., Chis., ca. San Cristóbal & & & $\mathrm{X}$ & $\mathrm{X}$ \\
\hline PLEOPELTIS & & & & & \\
\hline P. angusta var. stenoloma & 4352 (IZTA, UAMIZ); Méx., Oax., Valle Nal., ca. Esperanza & & & $\mathrm{X}$ & $\mathrm{X}$ \\
\hline P. fallax & 4347 (IZTA, UAMIZ); Méx., Pue., Cuetzalán, salto San Andrés & & & $\mathrm{X}$ & $\mathrm{X}$ \\
\hline P. mexicana & $\begin{array}{l}4334 \text { (IZTA, UAMIZ); Méx., Hgo, Zacualtipán, a } 2 \text { km desv. a } \\
\text { Tianguistengo }\end{array}$ & & & $\mathrm{X}$ & $\mathrm{X}$ \\
\hline SERPOCAULON & & & & & \\
\hline S. falcaria & $\begin{array}{l}4196 \text { (IZTA, UAMIZ); Méx., Pue., Zacapoaxtla, Apulco, salto La } \\
\text { Gloria }\end{array}$ & $\mathrm{X}$ & $\mathrm{X}$ & $\mathrm{X}$ & $\mathrm{X}$ \\
\hline & 4351 (IZTA, UMIZ); Méx., Oax., Valle Nacional, ca. Esperanza & & & & $\mathrm{X}$ \\
\hline S. triseriale & 4186 (IZTA, UAMIZ); Méx., Ver., Catemaco, ca. Coyame & $\mathrm{X}$ & $\mathrm{X}$ & $\mathrm{X}$ & $\mathrm{X}$ \\
\hline SYNAMMIA & & & & & \\
\hline S. feuillei & Harvey s.n. (K); Chile, Talcuhano & $\mathrm{X}$ & & & \\
\hline & Levin 509 (P); Chile, sin localidad & & & & $\mathrm{X}$ \\
\hline
\end{tabular}

